\title{
PIV Adaptive Interrogation and Sampling with Image Projection applied to water sloshing
}

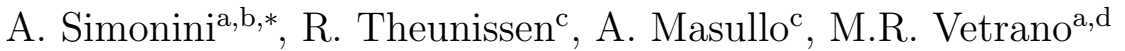 \\ ${ }^{a}$ von Karman Institute for Fluid Dynamics, Waterloosesteenweg 72, Sint-Genesius-Rode, \\ Belgium \\ ${ }^{b}$ TIPs Laboratory, Université Libre de Bruxelles, CP 165/67 50 av. F.D. Roosvelt, 1050 \\ Bruxelles, Belgium \\ ${ }^{c}$ Department of Aerospace Engineering, University of Bristol, University Walk, BS81TR, \\ Bristol, UK \\ ${ }^{d}$ Department of Mechanical Engineering, KU Leuven, Celestijnenlaan 300A, B-3001 \\ Heverlee, Belgium
}

\section{Abstract}

The measurement of fluid velocity in the vicinity of wavy interfaces by means of Particle Image Velocimetry (PIV) still constitutes a challenge. Besides the experimental complexities such as appropriate seeding, reflections due to gradients in refractive indices, aberrations, etc., also the image processing phase constitutes a critical component. Ignoring bias errors introduced by laser reflections near the interface, strong velocity gradients are typically encountered near the curved liquid/gas interface and are detrimental to the common cross-correlation analysis. These effects are exacerbated by the use of traditional rectangular static cross-correlation windows. Moreover, dynamic boundaries suffer from a certain loss in reliability due to the difference in number of particles located in a specific physical region between the two frames. In this paper we present an improved algorithm enhancing

\footnotetext{
*Corresponding author

Email address: simonini@vki.ac.be (A. Simonini)
} 
the accuracy of the velocity vector detection in dynamic wavy flows. This algorithm divides the fluid domain in subregions (i.e. bulk, intermediate, vicinity and interface) and applies to each region different features (such as image patching, window relocation and forward difference finite schemes) and ad-hoc predictors. Its assessment is performed on the basis of synthetic images, which reproduce a wavy motion similar to the one encountered when fluid in a cylindrical reservoir undergoes sloshing. When juxtaposed with a standard PIV code, numerical errors are shown to strongly diminish while improving the spatial resolution approaching the interface. The algorithm is finally applied to experimental PIV images of water sloshing in a cylindrical reservoir to provide more reliable results at the interface, contrary to standard PIV codes.

Keywords: Sloshing, PIV, Dynamic Interfaces

\section{Introduction}

Fluid sloshing is defined as the motion of the free liquid surface inside its container and it is strongly affected by external excitation applied to the partially filled container [? ]. The most well known example of sloshing is that of spilling coffee in a cup when walking [? ]. The understanding and prediction of this particular motion is of special importance in many fields. Indeed, the motion of liquids inside containers can refer to embarked containers filled with fluid, motion of cooling liquids in systems subjected to earthquake or motion of propellant inside the tank. The latter, for example, can influence the dynamic stability and performance of the vehicle, be it a ship, rocket or satellite. Although analytic solutions exist to describe the 
sloshing phenomenon, the underlying assumptions break down when sloshing amplitudes become too large due to inherent non-linearity [? ]. Numerical simulations have been performed, but these lack proper validation as a direct result of inaccurate experimental data near the liquid/gas interfaces [? ]. In particular, despite various studies investigating sloshing phenomena [? ], accurate data regarding the fluid velocity near the interface remains often absent.

In view of capturing both interface and bulk fluid dynamics simultaneously and instantaneously, the optical technique Particle Image Velocimetry (PIV) presents itself as most conducive metrology. Particle Image Velocimetry (PIV) has been applied to a wide variety of experimental fluid dynamics problems and underlying image analysis processes have seen great advancement. Standard image interrogation involves the division of image recordings into sub-sections (i.e. the interrogation or cross-correlation windows), in which a most probable particle image displacement is retrieved by means of cross-correlation. After removing unphysical displacement estimates [? ? ], images are subsequently deformed pixel-wise according to an interpolation of the estimated flow motion. The latter can be a filtered version of the crosscorrelation results in an attempt to minimize inherent modulation artifacts [? ]. The process is repeated in a recursive manner whereby interrogation areas are reduced to maximize spatial resolution. Despite small variations, the described process generally forms the backbone of each PIV image analysis algorithm [? ]. However, in the vicinity of static interfaces no consensus is reached as to which is the appropriate image interrogation procedure to maximize accuracy [? ]. This problem is exacerbated when dealing with dynamic 
interfaces and in particular liquid sloshing, which is the focus of the current research. Moreover, strong velocity gradients are introduced in the flow as particle images near the interface move in harmony with the free surface [? ] while this motion is dampened out with increasing distance from the interface. For this reason an alternative image analysis routine is presented in the following, providing supportive arguments to the chosen procedures. In our work an amalgamation of existing image processing routines is presented to enable accurate measurement both near and away from the liquid interface. As will be shown, the method proposed circumvents the problematic of ambiguity in the coordinate transformation by allowing the curvilinear system from one snapshot to be rearranged during the image deformation sequence. In addition, spatially varying interrogation parameters in terms of correlation window size and vector spacing is imposed to enhance the spatial resolution [?].

The paper is composed by 5 sections including the Introduction. Sec. 2 describes the impact that the presence of a dynamic interface has on the flow velocity measurements by PIV and presents several strategies reported in literature. Sec.3 presents the improved PIV algorithm, combining adaptive image sampling and sequential image analysis. This algorithm is assessed on the basis of synthetic images in Sec. 4, compared to a standard PIV algorithm, and subsequently applied to experimental sloshing images in Sec. 5. Finally in Sec. 6 conclusions are drawn and main outcomes are reported. 


\section{Problem statement: PIV near dynamic interfaces}

The first problem that a standard PIV algorithm encounters when dealing with dynamic interfaces with large amplitudes is the loss of correspondence between interrogation areas overlapping the boundaries. To exemplify, consider an interrogation area which, at time $t_{1}$ contains a portion of the interface (Fig. 1). If the amplitude of the interface displacement is larger than the window size, there will be a time $t_{2}=t_{1}+\Delta t(\Delta t$ represents the separation time between consecutive image recordings) at which this interrogation area captures a portion of either the liquid or gas phase. Conversely, there are also time instants $t_{n}$ at which the interrogation area would contain an insufficient number of particle images to retrieve a reliable displacement estimate [? ]. Such effects can be mitigated by selecting a sufficiently small separation time $\Delta t$, thus reducing the interface amplitude. However, choosing a very small $\Delta t$ compromises the achievable measurement accuracy in the bulk, where the motion is small, as a direct result of the random error in pixel displacement inherent to image processing. This random error is typically in the order of 0.01 pixels [? ].

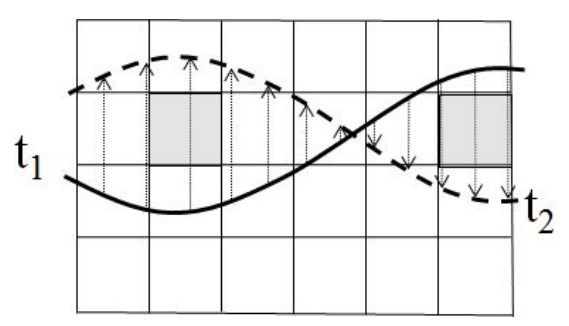

Figure 1: In-plane loss of particles due to a displacement of the interface larger than the windows size (grey window).

Irrespective of interface motion, depending on the degree of overlap be- 
tween the correlation windows and the interface, the interrogation area may generally contain an insufficient amount of tracer images leading again to a loss in reliability in the retrieved correlation results. This problem can be solved, in specific cases and in absence of large velocity gradients across the interface, by seeding both phases when dealing with gas-liquid interfaces as is common procedure when investigating e.g. surface waves [? ]. It should be noted that this does not solve the problem of loss of consistency in captured flow region (Fig. 1). Alternatively, more complex image processing algorithms can be employed. Tsuei at al. [? ] directly modify image recordings by mirroring image intensities across the interfaces for which the velocity must be known. For sloshing applications an iterative process was described to estimate the a-priori unknown interface motion. The latter is calculated in a first iteration through bi-linear interpolation of the velocity values obtained by cross-correlation at about a window size below the interface. This procedure is however unreliable since it is based on cross-correlation estimates in the near vicinity of the interface, which is unreliable for the above mentioned reasons. Theunissen et al. [? ] presented a method in which interrogation windows are forced to be aligned and stretched parallel to the identified boundaries, thus allowing the loss of particle image pairs to be minimised. This method is restricted however to stationary interfaces. For dynamic boundaries Jeon and Sung [? ] describe a methodology, inspired by the work of Nguyen et al. [? ], whereby image regions in the near vicinity of the interface are transformed along fitted curvilinear coordinates, thus rendering the interface into a straight line in the transformed image. In these pseudo-images conventional image processing algorithms can be used and 
displacement gradients near the interface are estimated using a gradiometry method proposed by Nguyen and Wells [? ]. The obtained displacement estimates are subsequently transferred back into the original Cartesian reference frame by inverting the curvilinear coordinates system. A similar processing has also been applied in the work of Löhrer et al. [? ] where a unique body conform transformation is assigned to each snapshot in order to produce surface aligned images.

In general the necessary conversion of the displacements obtained by means of correlation in the pseudo-image reference frame $(\xi, \eta)$ to the original coordinate system $(\mathrm{x}, \mathrm{y})$ will involve a Jacobian matrix containing the derivative terms of the mapping functions. While this is a valid approach when both image snapshots are subjected to the same transformation, the dynamic interface evolution will inhibit the use of a unique coordinate mapping; an interrogation grid conform to the interface at time instant $t_{1}$ will not necessarily fit the interface at $t_{2}$ and is therefore unsuitable for image analysis in close vicinity of the interface (Fig. 2).

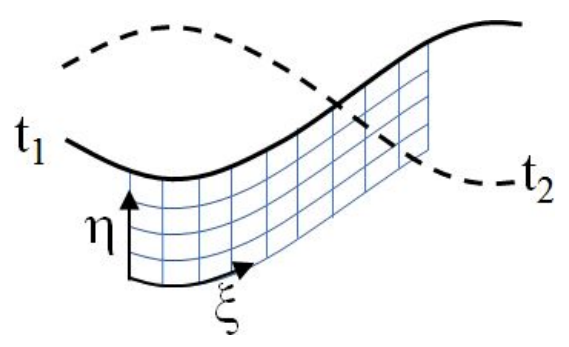

Figure 2: A curvilinear coordinate system fitted to the interface at one single time instant may no longer be appropriate when dealing with large interface displacement amplitudes.

Reducing the time separation between image recordings to yield sufficiently small discrepancies between interfaces, and therefore allowing to use 
an unique curvilinear coordinate system, has however been argued not to be generally adequate. Ideally, each snapshot should therefore have an individual numerical mesh and an associated Jacobian. This however raises the question wich of the curvilinear coordinate systems should be used to perform the cross-correlation and which re-scaling, related to the Jacobian of the adopted reference curvilinear grid system, should be applied to the obtained displacement vectors. This ambiguity hampers the use of multiple curvilinear meshes. Consequently, methods aimed at improving spatial resolution such as the adaptive stretching of particle images by Jia et al. [? ] are no longer applicable.

Not only the potentially large displacement of the interface but also the disagreement between the regularly spaced pixel coordinates and curved interface boundaries have been identified as problematic for the retrieval of particle image displacements when adopting pixel-defined correlation windows. Li et al. [? ] and Park et al. [? ] showed that in case of marginally different boundaries, the transformation of images by simply vertically shifting image pixels is able to improve correlation reliability. The present authors have scrutinised the generality of this methodology in the Appendix. It is shown that this approach introduces non-negligible particle image deformations when dealing with larger interface dynamics and more importantly, artificial velocity gradients. While the influence of particle image distortions can be minimized by means of adequate sub-pixel fitting schemes [? ] or image resampling [? ], the introduction of strong artificial displacement gradients and curvature (depending on the spatial variation in vertical shift) directly influence the reliability of the cross-correlation and deteriorate mea- 
surement accuracy [? ].

\section{PIV adaptive interrogation and sampling for large amplitude waves with image projection.}

The analysis of PIV image recordings pertaining large interface motions is faced with multiple issues. First, sufficient spatial resolution is needed in the close vicinity of the interface to resolve higher flow gradients. Second, the curved interfaces necessitate aligned correlation windows as to minimize overlap and maximize correlation reliability. However, the use of distinct curvilinear meshes in both snapshots is impeded by the ambiguity in obtained results. Third, Masullo and Theunissen [? ] argued the concept of image mapping in PIV, which is inherent to the use of curvilinear grids, to be beneficial in terms of accuracy on the condition that flow gradients are limited. Especially non-linear sloshing is characterized by strong gradients near the interface however. Together with the non-negligible interface motion between image snapshots, this inhibits the calculation of displacements using traditional image evaluation processes. Instead, in this section the authors present a sequential image interrogation routine acronymed PAISSIIP; PIV

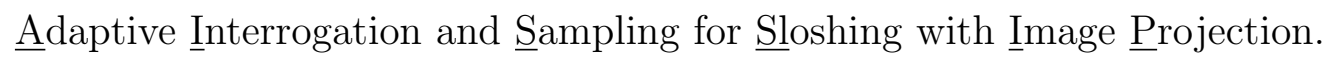

\subsection{Interface adaptive image sampling}

Nogueira et al. [? ] argued vector spacing to be one of the limiting factors in the spatial resolution achievable with PIV. With minor displacements in the bulk of the fluid and stronger gradients near the interface, an augmented vector density near the liquid surface is considered beneficial. This has been achieved by spatially altering the degree of overlap, WOR, between 
the correlation windows, as well as their spatial extent through a window size refinement factor, R. In both cases the spatial variation imposed is related to a scaled Wendland function (eq. 16);

$$
\begin{array}{r}
W O R(r)=\left(1-W_{0}\right)+\left(2 \cdot W O R_{0}-1\right) \cdot \varphi(r, \epsilon) \\
R(r)=1+\left(R_{0}-1\right) \cdot \varphi(r, \epsilon) \\
W_{s}(r)=R(r) \cdot W_{s}^{k}
\end{array}
$$

The Wendland function has been chosen as it is a continuous function resembling a Gaussian [? ], yet with finite extent and well-defined derivatives. As the interface dynamics are predominantly in vertical direction, the radial distance $r(x)$ is simply taken as the vertical distance from the detected interface, located at $y_{\text {int }}(x)$ at time $t_{1} ; r(x)=\left|y-y_{\text {int }}(x)\right|$. The extent $\epsilon$ of the Wendland function should allow a gradual variation in window positions. The authors have found $\epsilon=\left(8 \cdot W_{S}^{k}\right)-1$ to be suitable in all cases tested (superscript $\mathrm{k}$ refers to the window refinement iteration). Correlation window sizes and mutual overlap remain invariant along the x-direction and a constant WOR of $50 \%$ along the horizontal axis has been imposed, thus fixing the horizontal vector spacing to $h_{x}=0.5 \cdot W S_{k}$. Parameters $W O R_{0}$ and $R_{0}$ denote the imposed window overlap ratio and refinement at the interface, i.e. given a global interrogation window size in the $k^{t h}$ iteration, $W_{S}^{k}$, the correlation window size at the interface will equal $R_{0} \cdot W_{S}^{k}$. Due to the small dynamic velocity range in the bulk of the fluid an overlap ratio of $25 \%$ is sufficient whereas a smaller vector spacing is preferable near the interface. This translates into a $W O R_{0}=0.75$.

For each $x$, the location $y=y_{o}$ of the correlation window nearest to the interface overlapping the seeded liquid phase by a ratio $O$ is sought (Fig. 3a). 


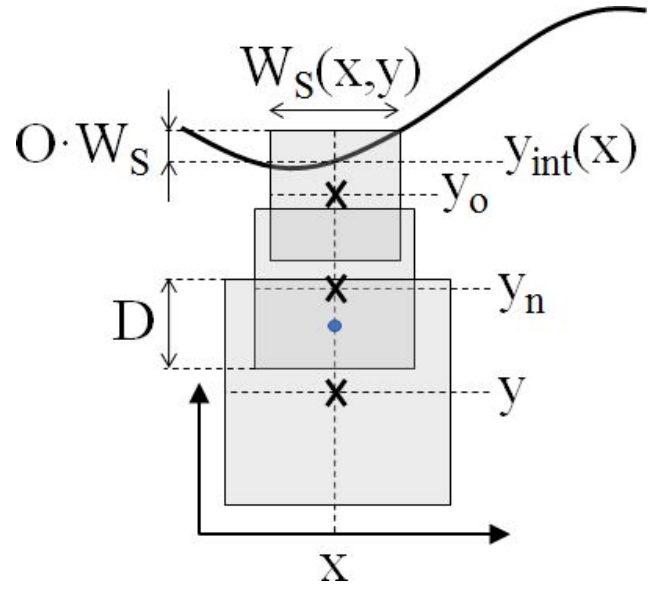

(a)

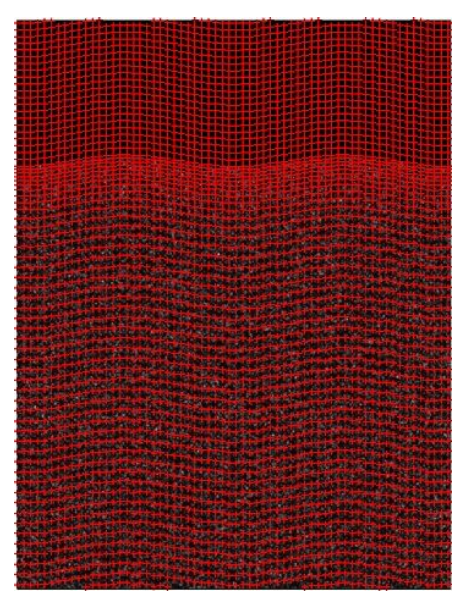

(b)

Figure 3: : (a) Parameter definition in the adaptive positioning and sizing of correlation windows for a given $\mathrm{x}$-location. Crosses indicate the centroids of the correlation windows. (b) Refined window distribution near an oscillating interface.

This location is found through the equality $y+0.5 \cdot W_{S}\left(\left|y-y_{\text {int }}\right|\right)-y_{\text {int }}=$ $O \cdot W_{S}\left(\left|y-y_{\text {int }}\right|\right)$ and will subsequently be a root of $f(y)$ defined as

$$
\begin{aligned}
f(y) & =\left(y-y_{\text {int }}(x)\right)+\left(\frac{1}{2}-O\right) \cdot W_{S}\left(\left|y-y_{\text {int }}(x)\right|\right) \\
\frac{d f(y)}{d y} & =1+\left(\frac{1}{2}-O\right) \cdot W_{S}^{k} \cdot\left(\frac{d R(|r|))}{d y}\right. \\
& =1+\left(\frac{1}{2}-O\right) \cdot W_{S}^{k} \cdot\left(R_{0}-1\right) \cdot \frac{\varphi(|r|, \epsilon)}{d|r|} \frac{d|r|}{d y}
\end{aligned}
$$

which can be found through a Newton-Raphson scheme adopting the initial solution $y_{o}=y_{\text {int }}(x)-0.5 \cdot W_{S}^{k}$ and derivative terms above with $d|r| / d y=$ $\operatorname{sgn}(r)$. The allowable overlap $O$ should be a compromise between being able to retrieve reliable correlation estimates while minimizing the radial distance. The authors found empirically a value of $60 \%$ to be appropriate. The following correlation window locations $\left(y<y_{\text {int }}\right)$ can again be retrieved 
through simple Newton-Raphson iterations. Having obtained the previous correlation window location $y_{n}$, the local overlap $D$ and average overlap ratio $W O R_{a v g}$ (see Fig. 3a) are given by

$$
\begin{gathered}
D=\left\{y_{n}-\frac{1}{2} W_{S}\left(y_{n}-y_{\text {int }}(x)\right)\right\}+\left\{y+\frac{1}{2} W_{S}\left(y-y_{\text {int }}(x)\right)\right\} \\
W O R_{\text {avg }}=\frac{1}{2}\left(\frac{D}{W_{S}\left(\left|y_{n}-y_{\text {int }}(x)\right|\right)}+\frac{D}{W_{S}\left(\left|y-y_{\text {int }}(x)\right|\right)}\right)
\end{gathered}
$$

Equating $W O R_{a v g}$ to the overlap defined midway between two consecutive correlation windows, i.e. $\operatorname{WOR} R_{\text {avg }}=\operatorname{WOR}\left(\frac{1}{2}\left(y-y_{\text {int }}(x)+y_{n}-y_{\text {int }}(x)\right)\right)$, yields the final equation of which the derivative can be expressed in terms of $d \varphi / d r$ and $\operatorname{sgn}(r)$ using Eq. 1;

$$
\begin{aligned}
& f(y)=2 \cdot \operatorname{WOR}\left(\frac{1}{2}\left(y+y_{n}\right)-y_{\text {int }}(x)\right) \cdot W_{S}\left(\left|y-y_{\text {int }}(x)\right|\right) \cdot W_{S}\left(\left|y_{n}-y_{\text {int }}(x)\right|\right) \\
&-\left\{\left(y_{n}-\frac{1}{2} W_{S}\left(\left|y_{n}-y_{\text {int }}(x)\right|\right)\right)-\left(y+\frac{1}{2} W_{S}\left(\left|y-y_{\text {int }}(x)\right|\right)\right)\right\} \\
&\left.\left.\cdot\left\{W_{S}\left(\left|y_{n}-y_{\text {int }}(x)\right|\right)\right)+W_{S}\left(\left|y-y_{\text {int }}(x)\right|\right)\right)\right\}
\end{aligned}
$$

The final window positioning illustrated in Fig. 3b shows a concentration of correlation window centers near the interface, with increasingly larger separation in the vertical direction away from the interface.

\subsection{Sequential image analysis}

To minimize the effect of gradients in the near vicinity of the interface when estimating displacements, image analysis is sequenced. The process is graphically depicted in Fig. 4. The first domain concerns the bulk of the flow and contains all correlation windows below $y_{\min }(x)$, the location of the correlation window nearest to the interface in each column for which no overlap 
with the interface is present in either of the image snapshots. Correlation windows lying above $y_{\min }(x)$ will overlap with the interface to a certain extent and are considered susceptible to generating outliers. To account for the interface displacement and increase the robustness of the correlation, in the very first iteration corresponding correlation windows are translated vertically in the second image snapshot according to the detected shift in surface at a specific $x$ location; $y\left(x, t_{2}\right)=y\left(x, t_{1}\right)+\Delta y_{\text {int }}(x) \varphi\left(\left|y-y_{\text {int }}\right|, \epsilon_{\text {int }}\right)$ where $\Delta y_{\text {int }}(x)=y_{\text {int }}\left(x, t_{2}\right)-y_{\text {int }}\left(x, t_{1}\right)$ and $\epsilon_{\text {int }}=\left(8 \cdot \Delta y_{\text {int }}(x)\right)^{-1}$. Conform to standard interrogation processes, correlation windows are recursively refined (i.e. halved in size) and image deformation is applied to the second image snapshot after vector validation. Note that the adopted shift of the correlation windows is used to update only the displacement estimates in the first iteration. The intermediate image region spanning from the interface to the limit of the bulk flow is analysed next, ignoring correlation windows overlapping the interface by more than typically $60 \%$. Here extrapolation of the displacement field obtained in step 1 serves as input for image deformation and the recursive process involving window refinement and deformation is repeated. Standard procedure is followed in the correlation of the involved windows. To negate the influence of the non-seeded area in case correlation windows overlap the interface and to adequately attribute obtained crosscorrelation displacement vectors, the mean intensity of the enclosed seeded region is patched into the non-seeded area and the vector is attributed to the center of gravity of the seeded area $([?])$. The third region concerns the near vicinity of the interface where strong gradients are expected. This region is therefore more prone to the occurrence of erroneous vectors, despite 
vector validation, augmenting the uncertainty in obtained data. To reduce underlying flow gradients, displacements from the intermediate region are extrapolated and combined into predictors $\Delta x$ and $\Delta y$ to initially deform this region. In addition, correlation windows are aligned with the detected interface geometry in the first of the image pair and relocated in the second snapshot. Note that if the predictors have accurately quantified the particle displacements, the relocated curvilinear grid will be conform to the interface at time $t_{2}$. This will be explained in further detail in section 3.4. To further accommodate stronger gradients parallel to the interface and enhance spatial resolution, non-isotropic windows are imposed with reduced extent normal $W_{S, i}$ (typically in the order of 7 to 13 pixels) and $50 \%$ mutual overlap. Two iterations are applied in the near vicinity, halving the extent of the correlation window parallel to the interface. The entire process is finally repeated utilizing an initial window size twice the final interrogation dimensions to further minimize any residual displacement. Finally, the image area between the interface boundary and first curvilinear vector (located at a vertical distance $W_{S, i}$ from the interface) is analyzed using a method reminiscent of the interface gradient method described by Jeong and Sung [? ], iteratively decreasing the vector spacing (see section 3.4).

\subsection{Image projection}

Based on the interface position at time $t_{1}$ a curvilinear mesh $(\xi, \eta)$ is created (Fig.5). With displacement predictors $\Delta x(x, y)$ and $\Delta y(x, y)$ the pixel locations at time $t_{2}$ are predicted. The curvilinear reference system $(\xi, \eta)$ remains therefore unchanged throughout the analysis; it is the transformation by $\Delta x$ and $\Delta y$ guiding the location of the curvilinear coordinate system 


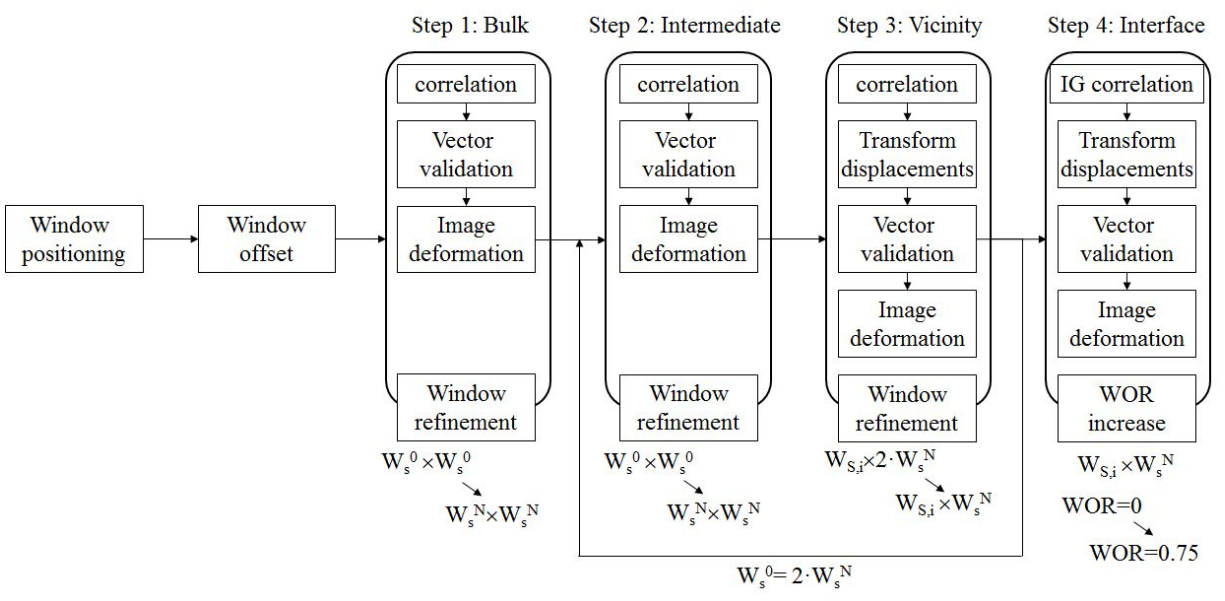

Figure 4: Layout of the adopted sequential image interrogation process.

in the second image frame in an attempt to tailor it to the corresponding interface by invoking the measured particle image displacement estimates. The suitability of the deformed curvilinear correlation window in the second snapshot is therefore dictated by the correctness of the displacement predictors $\Delta x$ and $\Delta y$, referring to the gradual minimization of the influence of flow gradients by sequential image interrogation.

The curvilinear coordinates can be expressed in terms of the rectangular coordinates $(x, y)$ and restricting image deformation to $I_{2}$ allows the images to be transformed into $I_{1}^{*}$ and $I_{2}^{*}$;

$$
\begin{aligned}
& I_{1}(x, y)=I_{1}((x(\xi, \eta), y(\xi, \eta)))=I_{1}^{*}(\xi, \eta) \\
& \begin{array}{l}
I_{2}(x+\Delta x, y+\Delta y)= \\
=I_{2}(x(\xi, \eta)+\Delta x(x(\xi, \eta), y(\xi, \eta)), y(\xi, \eta)+\Delta y(x(\xi, \eta), y(\xi, \eta)) \\
\quad=I_{2}(x(\xi, \eta)+\tilde{\Delta} x(\xi, \eta), y(\xi, \eta)+\tilde{\Delta} y(\xi, \eta))=I_{2}^{*}(\xi, \eta)
\end{array}
\end{aligned}
$$




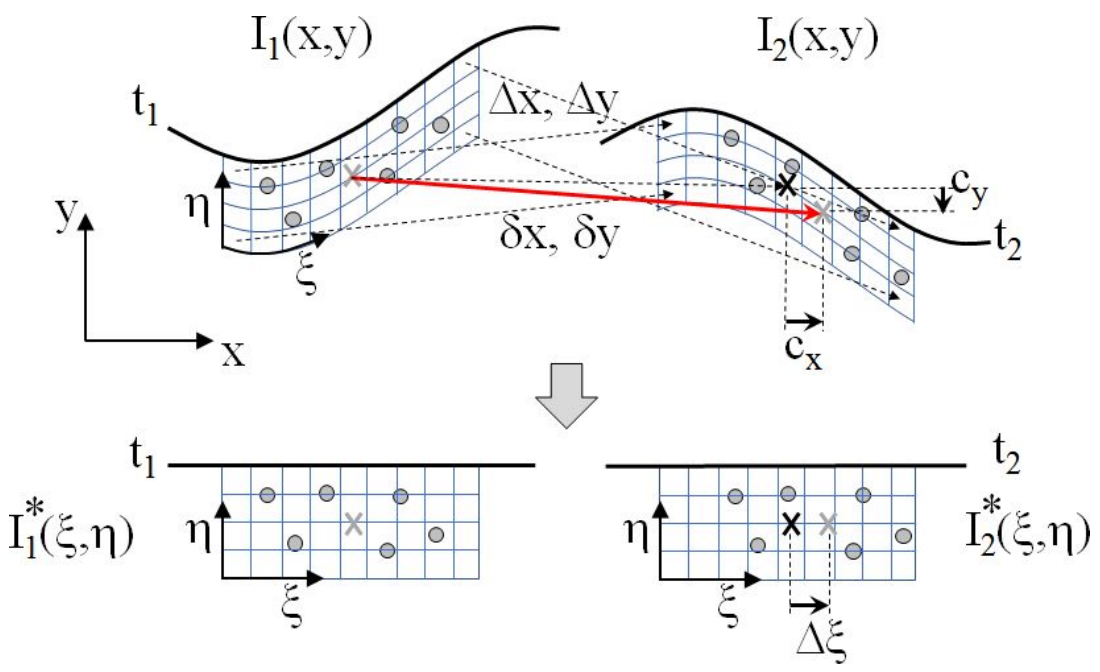

Figure 5: Projection of the curvilinear grid defined in $I_{1}$ to $I_{2}$ using displacement predictors $\Delta x$ and $\Delta y$. The actual shift of the particle image pattern is given by $\left(\delta_{x}, \delta_{y}\right)$ and indicated by the red arrow, whereas the centroid of the correlation window is represented by the black cross mark. Displacements $\Delta \xi$ and $\Delta \eta$ resulting from the correlation of transformed images are subsequently converted into $c_{x}$ and $c_{y}$, defining the corrected template shifts with respect to the correlation window centroid.

Correlation between the two images $I_{1}^{*}$ and $I_{2}^{*}$ will result in displacements $\Delta \xi$ and $\Delta \eta$, quantifying the required shift of the second interrogation area to have an identical particle image pattern in the corresponding interrogation area in $I_{1}$. It is important to note that only one curvilinear coordinate system is adopted removing any ambiguity as to which reference frame the correlation pertains. With Eq.7 the obtained shift can be expressed in the $(x, y)$ reference frame;

$$
\begin{aligned}
{[x(\xi+\Delta \xi, \eta+\Delta \eta)} & +\tilde{\Delta} x(\xi+\Delta \xi, \eta+\Delta \eta)]-[x(\xi, \eta)+\tilde{\Delta} x(\xi, \eta)]= \\
& =\frac{\partial x}{\partial \xi} \Delta \xi+\frac{\partial x}{\partial \eta} \Delta \eta+\frac{\partial \tilde{\Delta} x}{\partial \xi} \Delta \xi+\frac{\partial \tilde{\Delta} x}{\partial \eta} \Delta \eta+O\left(\Delta^{2}\right)
\end{aligned}
$$


A similar procedure in the $y$-direction allows the shift in window centroid to be expressed as

$$
\left[\begin{array}{l}
c_{x} \\
c_{y}
\end{array}\right]=\left[\begin{array}{ll}
\frac{\partial x}{\partial \xi}+\frac{\partial \tilde{\Delta} x}{\partial \xi} & \frac{\partial x}{\partial \eta}+\frac{\partial \tilde{\Delta} x}{\partial \eta} \\
\frac{\partial y}{\partial \xi}+\frac{\partial \tilde{\Delta} y}{\partial \xi} & \frac{\partial y}{\partial \eta}+\frac{\partial \tilde{\Delta} y}{\partial \eta}
\end{array}\right]\left[\begin{array}{c}
\Delta \xi \\
\Delta \eta
\end{array}\right]
$$

In the current work the curvilinear coordinate system is aligned with the identified interface boundary, $y_{\text {int }}\left(x, t_{1}\right)$, by imposing a mere vertical, uniform, pixel translation columnwise: $\xi(x, y)=\xi(x)=x$ and $\eta(x, y)=y-y_{\text {int }}(x)$, simplifying the transformation in 9 to

$$
\left[\begin{array}{l}
c_{x} \\
c_{y}
\end{array}\right]=\left[\begin{array}{cc}
1+\frac{\partial \tilde{\Delta} x}{\partial \xi} & \frac{\partial \tilde{\Delta} x}{\partial \eta} \\
\frac{\partial y_{i n t}}{\partial x}+\frac{\partial \tilde{\Delta} y}{\partial \xi} & 1+\frac{\partial \tilde{\Delta} y}{\partial \eta}
\end{array}\right]\left[\begin{array}{c}
\Delta \xi \\
\Delta \eta
\end{array}\right]
$$

Presuming momentarily a uniform shift of the related particle image pattern $I(x, y)$ by $(\delta x, \delta y)$ along the interface boundary; $I_{2}(x+\delta x, y+\delta y)=I(x+$ $\left.\delta x, y+\delta y, t_{2}\right)=I\left(x, y, t_{1}\right)=I_{1}(x, y)$, the estimated vector components at the centre of the correlation window are accordingly $\delta x_{e}=\frac{1}{W_{S}^{k}} \iint_{W_{S}^{k}} \Delta x(x, y) d x d y+$ $c_{x}$ and $\delta y_{e}=\frac{1}{W_{S}^{k}} \iint_{W_{S}^{k}} \Delta y(x, y) d x d y+c_{y}$ which serve as input for the subsequent iterative image deformation routine. In the current approach, a conventional forward difference (first order accurate) scheme has been adopted for simplicity, restricting image deformation to only one snapshot. Second order accurate difference schemes [? ] can be obtained by equally partitioning the correlation results. However, this requires the calculation of two Jacobians and adds complexity as it requires bookkeeping of two sets of displacements $\left(c_{x}, c_{y}\right)_{1}$ and $\left(c_{x}, c_{y}\right)_{2}$ for each of the snapshots respectively. More important, attributing only $\frac{1}{2} \Delta x$ and $\frac{1}{2} \Delta y$ to both images no longer ensures the curved interrogation area to comply with the interface at time $t_{2}$. In 
addition, the forward difference approach is in this case more intuitive (vectors relate to time instant $t_{1}$ and point towards the location of the particle images at time $t_{2}$ ). Ultimately second-order accuracy can still be achieved by a simple translation of a vector's initial point towards the half-way point or more elaborate schemes such as those presented by Scharnowski and Kähler $[?]$.

\subsection{Interface displacement}

While the application of a curvilinear grid as described in section 3.3 yields vectors distanced one vertical correlation window extent $\left(W_{S, i}\right)$ from the interface, the flow motion in the remaining image area can be resolved by means of a correlation based on imposing corrections on the estimated interface motion. Obtained displacements are extracted towards the interface, $u_{i, i}, v_{i, i}$, and for each correlation window $\left(W_{S, i} \times W_{S}^{N}\right.$ in size) corrections $c_{u}$ and $c_{v}$ are imposed ranging between $-\frac{1}{2} W_{S, i}$ and $+\frac{1}{2} W_{S, i}$ and $-\frac{1}{2} W_{S}^{n}$ and $+\frac{1}{2} W_{S}^{n}$ respectively (Fig. 6). For each combination of $c_{u}$ and $c_{v}$ the second image recording is deformed, based on a predictor $f$ utilizing the available and corrected displacements, and correlated in the curvilinear coordinate system (Fig. 6). The peak in the cross-correlation map $C\left(c_{u}, c_{v}\right)$ thus indicates the most appropriate correction on the horizontal and vertical interface displacements.

This method is an extended version of the IG correlation proposed by Jeon and Sung [? ] as for each velocity component the two orthonormal 


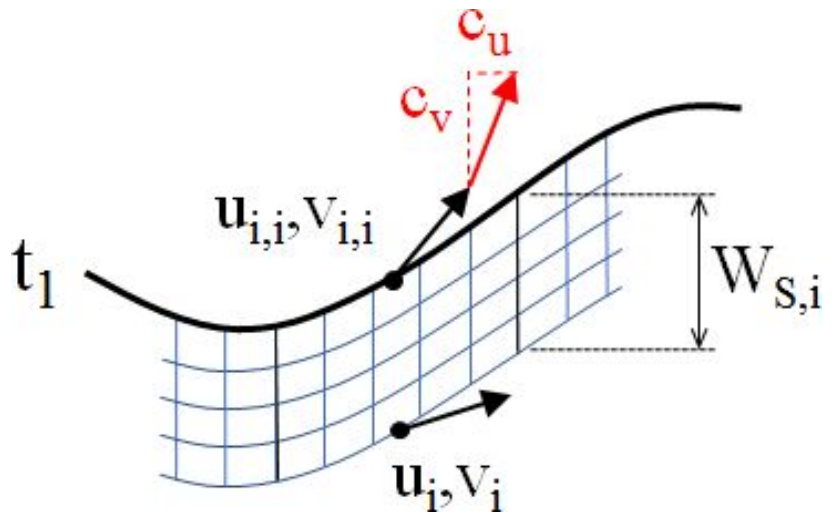

Figure 6: Principle of interface gradiometry whereby the interrogation window crosscorrelation is performed in function of corrections $c_{u}$ and $c_{v}$ superimposed on the displacements $u_{i, i} v_{i, i}$, which are obtained by extrapolating previous correlation results $u_{i}, v_{i}$ towards the interface.

gradients are considered through the repeated deformation of $I_{2}$;

$$
\begin{aligned}
C\left(c_{u}, c_{v}\right)=\sum_{n} & \sum_{m}\left(I_{1}^{*}(m, n)-\overline{I_{1}^{*}}\right) \\
& \left(I_{2}^{*}\left(m+f\left(u_{i, i}, u_{i}, c_{u}, c_{v}\right), n+f\left(v_{i, i}, v_{i}, c_{u}, c_{v}\right)\right)-\overline{I_{2}^{* *}}\right)
\end{aligned}
$$

with $\overline{I_{2}^{* *}}=\left(W_{S, i} W_{S}^{N}\right)^{-1} \sum_{n} \sum_{m} I_{2}^{*}\left(m+f\left(u_{i, i}, u_{i}, c_{u}, c_{v}\right), n+f\left(v_{i, i}, v_{i}, c_{u}, c_{v}\right)\right.$ The procedure is repeated, recursively increasing the mutual overlap between the single row of correlation windows from $0 \%$ to $75 \%$ (Fig. 4).

\section{Numerical assessment}

To assess the accuracy of the proposed PAISSIIP routine, time-resolved synthetic PIV images of a sinusoidal wavy flow were generated similarly to the sloshing linear theory described in [? ]. Images were $1000 \times 760 \mathrm{px}^{2}$ in size and were seeded with tracer particles randomly placed within the 
fluid with a concentration of $0.15 \mathrm{ppp}$ (particle per pixel). The pixel-wise integrated intensity of the particle images was modeled following a normal intensity distribution with a mean particle image diameter of 2 pixels, a standard deviation of 1 pixel and a pixel fill factor of 0.7 . For each image, Gaussian noise was added with mean intensity $5 \% I_{\max }$ and standard deviation $0.3 \% I_{\max }$, where $I_{\max }$ is the maximum intensity of the synthetic image.

At initial time $t_{0}=0 s$, the interface was defined to ly on a line at coordinate $y_{0}=C_{0}=750 p x$, corresponding to the initial height of the liquid/gas interface. The temporal evolution of each particle location along the $y$-direction has been related to the position of the interface at time $t_{0}$, combined with a spatial damping factor:

$$
y_{p}\left(x_{0}, y_{0}, t, t_{0}\right)=y_{0}-A_{Y} \cdot \cos \left(2 \pi \frac{x_{0}}{\lambda}\right) \cdot \sin \left(2 \pi \frac{t}{T}\right) \cdot\left(1-\tanh \left(\frac{y_{0}-C_{0}}{K_{1}}\right)\right)
$$

where $\left(x_{0}, y_{0}\right)$ are the initial particle coordinates at time instant $t_{0}$, the maximum displacement was given by $A_{Y}=50 \mathrm{px}$ with the sinusoidal period $T=1 / 3 s$, the sloshing wavelength $\lambda$ equalled $760 \mathrm{px}$ and parameter $K_{1}$ defines the extent of the damping factor in $y$ direction, which was set to 100 px. The particle motion along the $x$-abscissa was described as:

$$
\begin{aligned}
x_{p}\left(x_{0}, y_{0}, t, t_{0}\right)=x_{0}+ & A_{X} \cdot \sin \left(2 \pi \frac{t}{T}\right) \cdot \ldots \\
& \frac{1}{3}\left(\arctan \left(x_{0}\right)-\arctan \left(x_{0}-W\right)\right) \cdot G\left(\left|y_{0}-C_{0}\right|\right)
\end{aligned}
$$

where $W$ equals the width of the image (760 px), a limit of 20 px displacement was imposed $\left(A_{X}=20 \mathrm{px}\right)$ and term $\mathrm{G}$ takes into account the impact of 
damping on the horizontal velocity component;

$$
G(y)=1-\tanh \frac{y}{30}
$$

The term containing the difference between arctan functions was introduced to slow down the motion in $\mathrm{x}$-direction with the purpose of reducing the effect of escaping particles at the borders.

To incorporate the effect of varying interface curvature, Eqs.12 and 13 were utilized to determine the required $t_{a}=t$ corresponding to an initial interface with pre-defined amplitude at $x=\lambda / 2$. Exemplary images with time separations of $T / 8$ are presented in Fig. 7 .

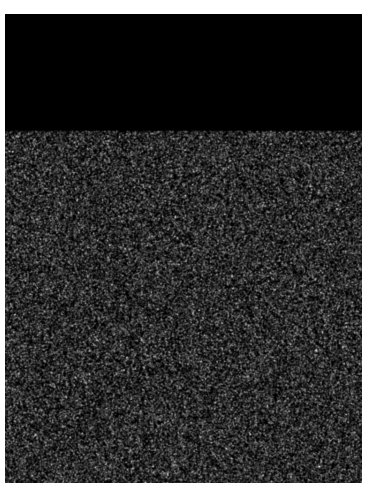

(a)

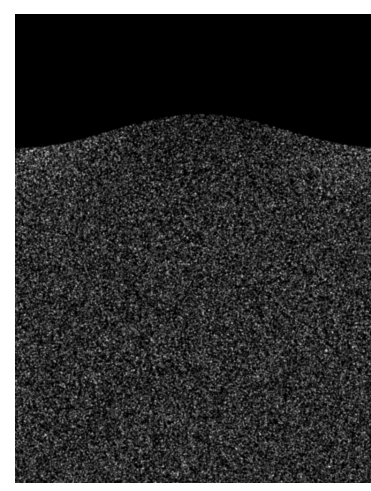

(b)

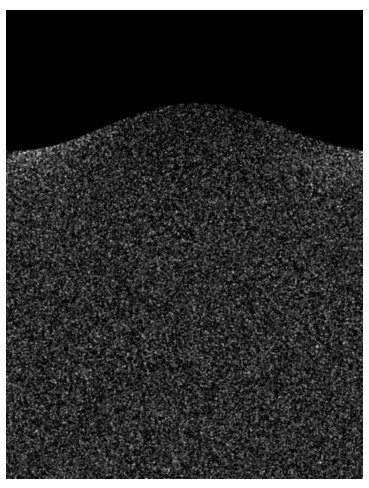

(c)

Figure 7: Synthetic PIV images (1000x760 $\left.\mathrm{px}^{2}\right)$ generated using the equations (12-14) with (a) $t=t_{0}=0$ (b) $t=T / 8$ (c) $t=T / 4$.

In order to assess the improvement on the PAISSIIP routine, a comparison with a standard PIV code incorporating window deformation adopting central differences and successive multigrid refinement [? ] has been performed. Six initial free surface conditions from a quasi-flat interface $\left(0.05 \cdot A_{Y}\right)$ to maximum amplitude $\left(A_{Y}\right)$ have been considered (table 1). For each initial 
Table 1: Initial $\left(t_{a}\right)$ and final time $\left(t_{\max }\right)$ adopted in the generation of the cases presented in Fig. 8. Variable $t_{\max }$ refers to the required time to yield a vertical interface motion of 16 pixels from the interface obtained at time $t_{a}$.

\begin{tabular}{ccccccc} 
& $0.05 \cdot A_{Y}$ & $0.2 \cdot A_{Y}$ & $0.4 \cdot A_{Y}$ & $0.6 \cdot A_{Y}$ & $0.8 \cdot A_{Y}$ & $1.0 \cdot A_{Y}$ \\
\cline { 2 - 7 }$t_{a}[\mathrm{~s}]$ & 0.0027 & 0.0107 & 0.0219 & 0.1326 & 0.1178 & 0.0775 \\
$t_{\max }[\mathrm{s}]$ & 0.0200 & 0.0289 & 0.0426 & 0.1515 & 0.1403 & 0.1275 \\
\hline \hline
\end{tabular}

surface amplitude, 16 time separations between successive snapshots were then selected to yield vertical displacements ranging from 1 to 16 pixels with steps of 1 pixel. The $6 \times 16$ cases are depicted in Fig. 8, while, in table 1 , the initial time $\left(t_{a}\right)$ to generate the initial interface and the final time $\left(t_{\max }\right)$ related to the maximum displacement of 16 pixels are reported for each case. The first three cases $\left(A=0.05 \cdot A_{Y}, A=0.2 \cdot A_{Y}, A=0.4 \cdot A_{Y}\right)$ have increasing curvature and positive (upward) interface motion, while the remaining cases have decreasing curvature and negative (downward) interface velocities. To extract statistical information in terms of displacement error, 100 instantaneous velocity maps were taken into account for each case.

The processing parameters are shown in table 2 . The synthetic flow is composed of a horizontal and a vertical sinusoidal motion (Eq. 13 and 12) such that the position of the maximum amplitude of the interface moves from $x=380 \mathrm{px}$ for the interface with amplitude $0.05 \cdot A_{Y}$, to $x=400 \mathrm{px}$ for the case where the amplitude equals $A_{Y}$. To provide a representative error estimate, the velocity errors in a region of 15 pixels below the interface and \pm 12 pixels in horizontal direction at the position where the interface displacement is maximum were averaged as illustrated in Fig. 9. Geometrical centres of the 

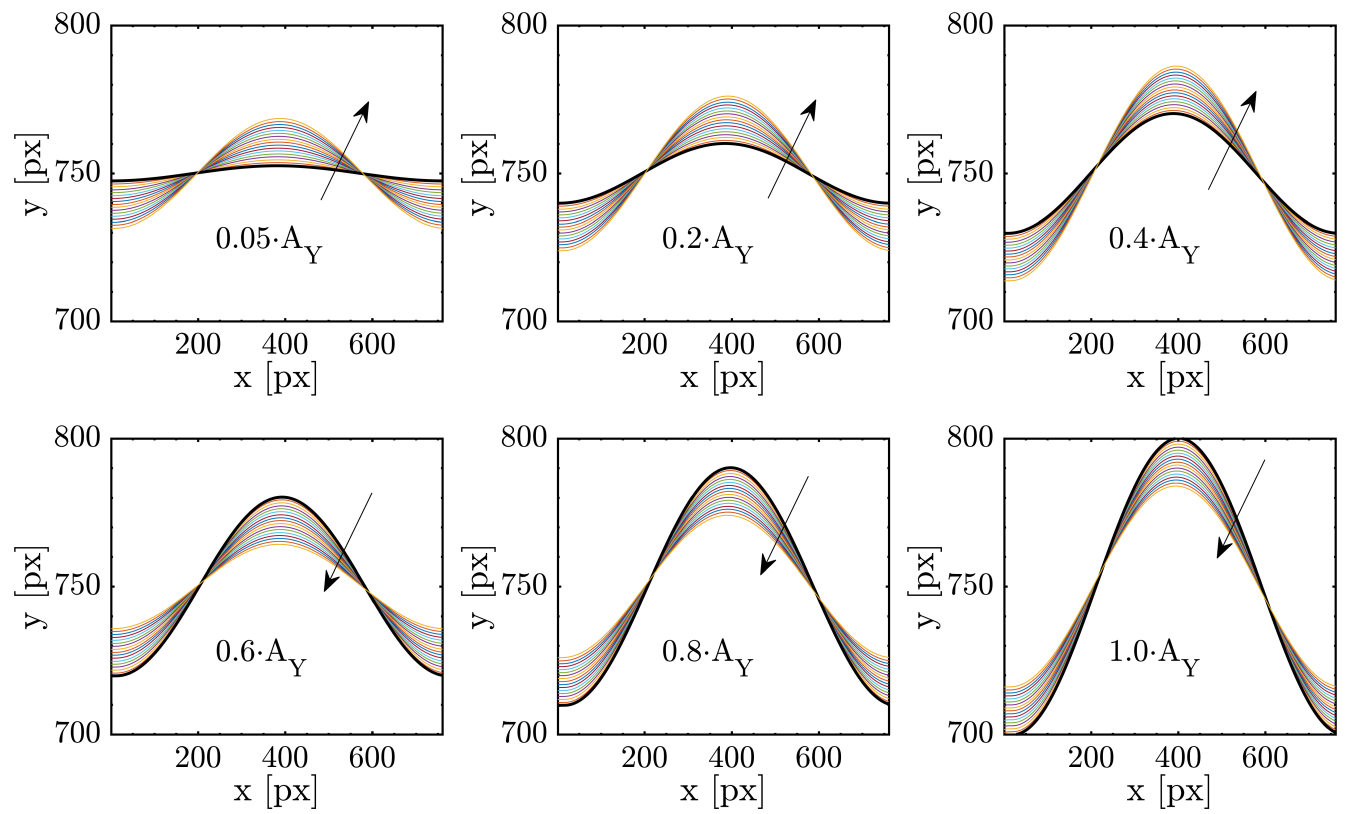

Figure 8: Illustration of the evolution in interface position considered in the numerical assessment. The amplitude of the initial interface at the first snapshot is shown in black and corresponds to the timing reported in Table 1. The other interfaces are generated imposing a displacements at the point of maximum curvature from 1 to 16 pixels with 1 pixel step. The arrow indicates the direction of the interface motion. 
Table 2: PIV processing parameters.

\begin{tabular}{|c|c|c|}
\hline & \\
\hline & PAISSIIP & Standard PIV \\
\hline Value & \multicolumn{2}{|c|}{ Quantity } \\
\hline Initial window size & $96 \times 96$ & $96 \times 96$ \\
\hline Number of refinement & 2 & 2 \\
\hline Window Overlap Ratio $(W O R)$ & $75 \%$ & $75 \%$ \\
\hline SN threshold ratio & 1.3 & 1.3 \\
\hline Maximum Interface $W O R_{\text {int }}$ & $60 \%$ & - \\
\hline Interface Vertical Window Size & 7 & - \\
\hline Adaptive Window Distribution & Wendland & - \\
\hline
\end{tabular}

windows obtained by the two routines considered are indicated considering the case where the amplitude equals $A_{Y}$ and maximum displacements of 14 pixels in the vertical direction were attained. This picture confirms PAISSIIP to allocate a higher vector density in the near-interface region with respect to a standard PIV code, allowing a higher resolution and better capture of velocity gradients. (One can observe in Fig. 9 that centroids of correlation windows allocated by the proposed routine can differ from the highlighted interface in the first snapshot by 1 pixel. This is due to the adopted line simplification algorithm for the interface boundary definition [? ]. Because of the implemented interface gradiometry approach (section 3.4) these inaccuracies do not degrade the overall algorithm performance.)

The mean discrepancies with respect to the synthetic flow have been calculated in the selected area together with the standard deviation over 100 


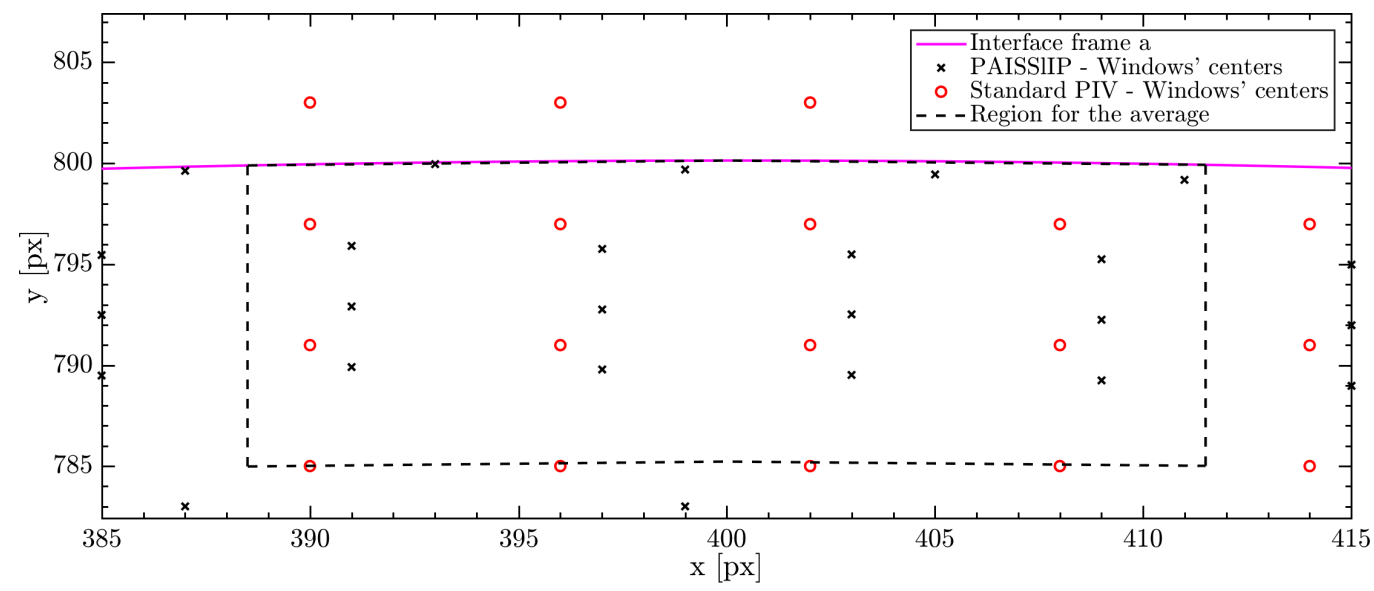

Figure 9: Illustration of correlation window placement in the near-vicinity of the fluid-gas interface for the case where the interface amplitude equals $A_{Y}$ and maximum horizontal displacements of 14 pixels are encountered. The interface detection is based on the first snapshot. The dashed rectangle indicates the region considered in the ensemble averaging for the numerical assessment.

calculated velocity maps and are displayed in Fig. 10. PAISSIIP presents mean errors along the horizontal direction which typically remain below 0.05 px and $0.2 \mathrm{px}$ in the vertical direction. The error remains nearly independent of the initial interface and a slight increase in error can be observed to a maximum of $0.18 \mathrm{px}$ in $\mathrm{y}$-direction and a standard deviation of $0.14 \mathrm{px}$ for larger displacements. The standard PIV approach on the other hand yields maximum errors of $2.35 \mathrm{px}$ with $0.69 \mathrm{px}$ deviation in y-direction. Clearly, PAISSIIP offers a big improvement compared to standard algorithms, especially in case of larger interface motion. A zoom on the PAISSIIP results is shown in Fig. 11. PAISSIIP consistently produces lower errors, especially for test cases corresponding to stronger interface curvatures. It can be observed that a standard PIV code has difficulties in estimating the proper horizontal 
velocity value towards the larger values of displacement. Contrary to the standard interrogation approach, PAISSIIP also shows to be insensitive to the change in curvature of the liquid/gas interface (positive y-displacement correspond to an increase of curvature while negative one to a decrease of curvature). This is due to both the selection of cross correlation windows during the first iteration and the use of forward differences.

An example of the spatial error distribution for both PAISSIIP and the standard PIV algorithm is shown in Fig. 12 for the case where the initial interface amplitude is $0.8 \cdot A_{Y}$ and maximum vertical displacements of 10 px are applied. The standard PIV code fails to yield reliable results in a large region below the interface for both velocity components returning errors in excess of $0.5 \mathrm{px}$. The alternative image analysis routines incorporated in PAISSIIP is able to reduce the error, yielding local maxima in the order of $0.2 \mathrm{px}$. The areas of higher error correspond to the region of increased velocity curvature due to the imposed damping (viz. $G$ in Eq.13) and image borders. Overall the regions of higher error are considerably reduced compared to straightforward correlation analysis and even at the position of maximum interface curvature errors are reduced.

Finally, a further assessment has been performed to demonstrate the variability of standard PIV results due to the dependence on the overlap between interface and correlation windows. To this extent the variable $C_{0}$, defining the initial location of the interface in the vertical direction at $t_{0}=0 \mathrm{~s}$, has been augmented by 2 and 3 pixels while maintaining identical processing parameters. The discrepancies with respect to the synthetic flow are shown in Fig. 13 for both codes. The error affecting the standard PIV approach is 

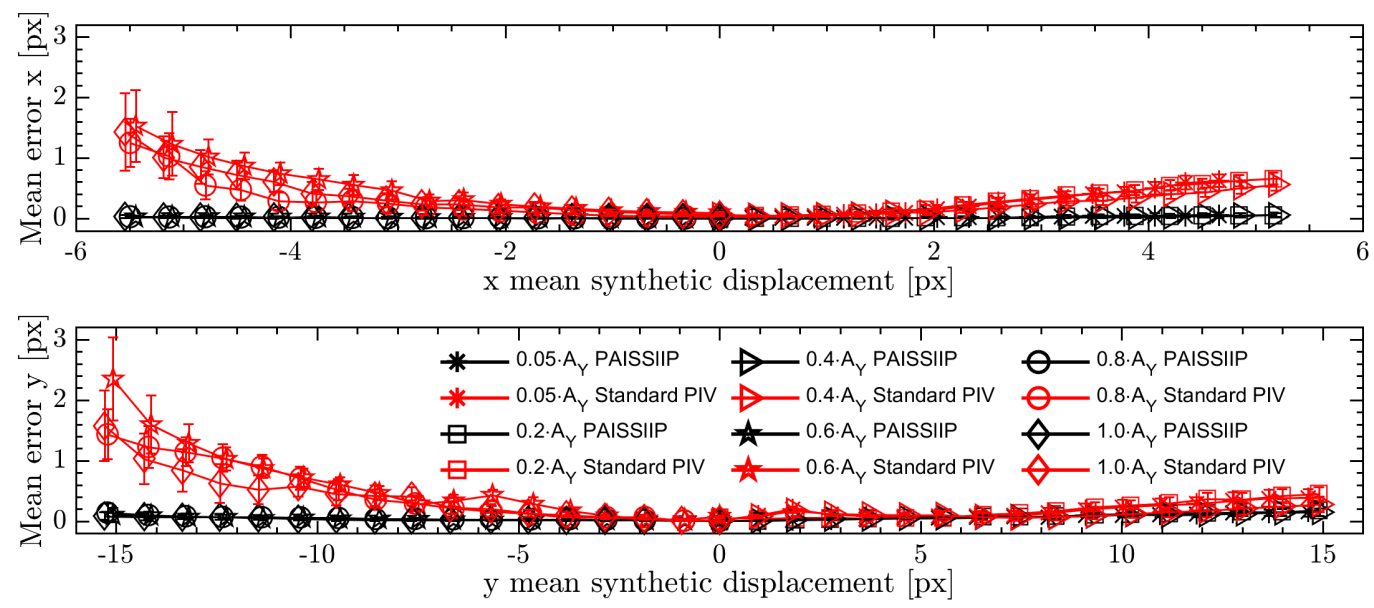

Figure 10: Mean discrepancies of the $\mathrm{x}$ and $\mathrm{y}$-displacements.
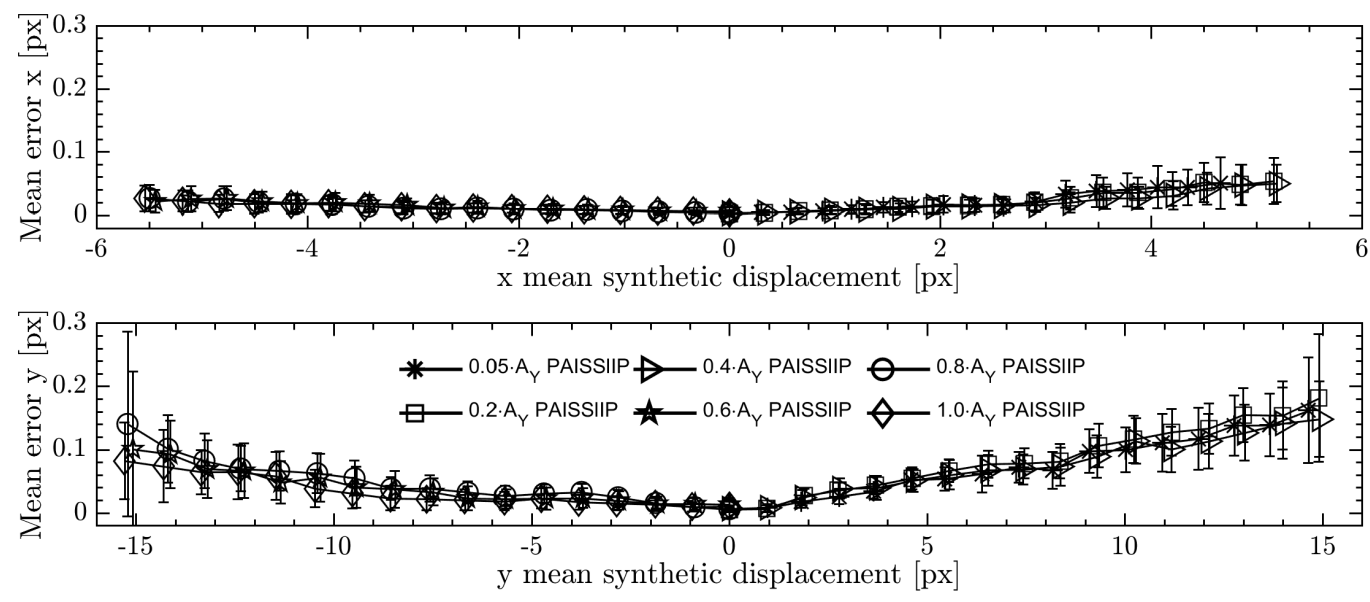

Figure 11: Mean discrepancies of the $\mathrm{x}$ and $\mathrm{y}$-displacements, zoom on PAISSIIP results. 


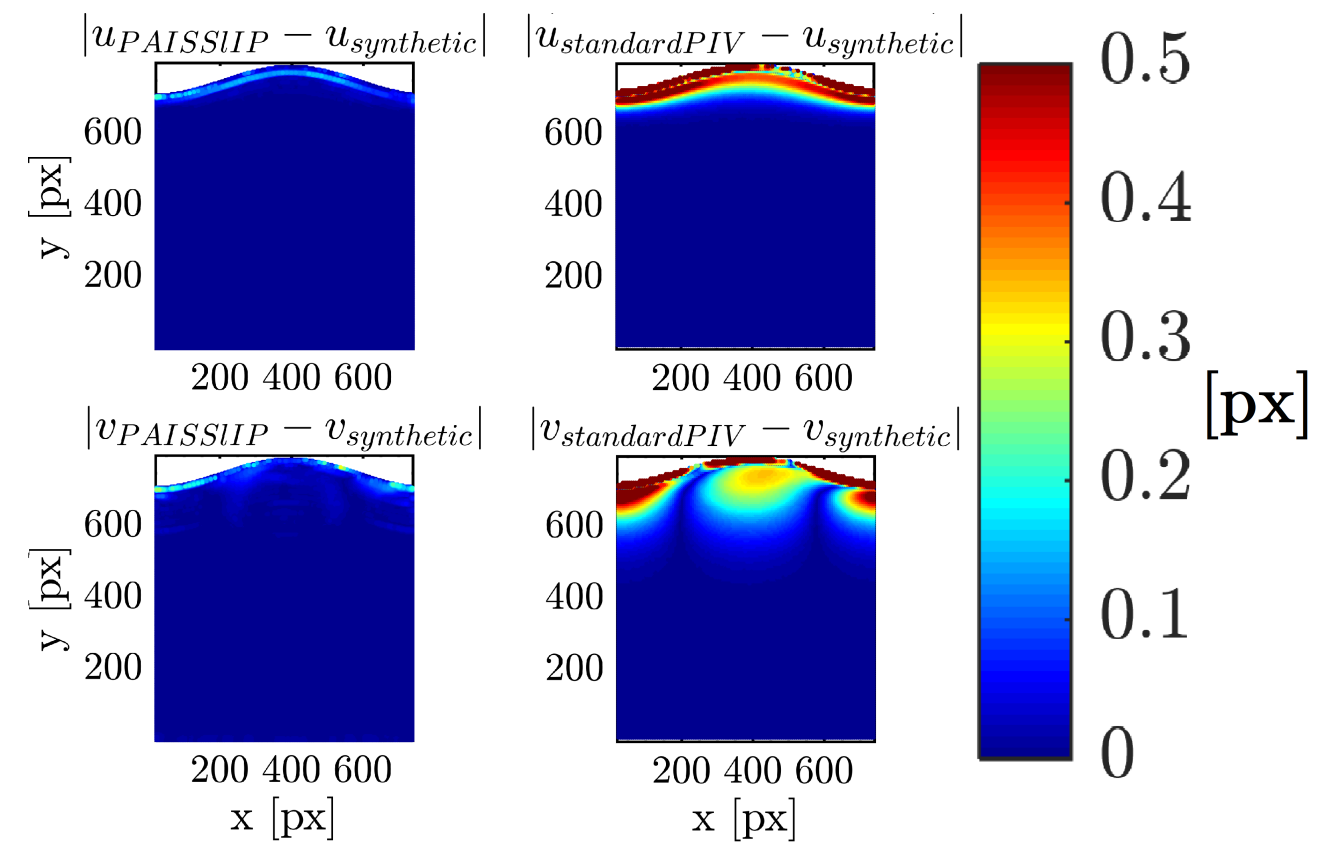

Figure 12: Mean discrepancies map of the $\mathrm{x}$ and $\mathrm{y}$-displacements for the synthetic test case with amplitude $0.8 \cdot A_{Y}$ and nominal displacement 10 pixels. 

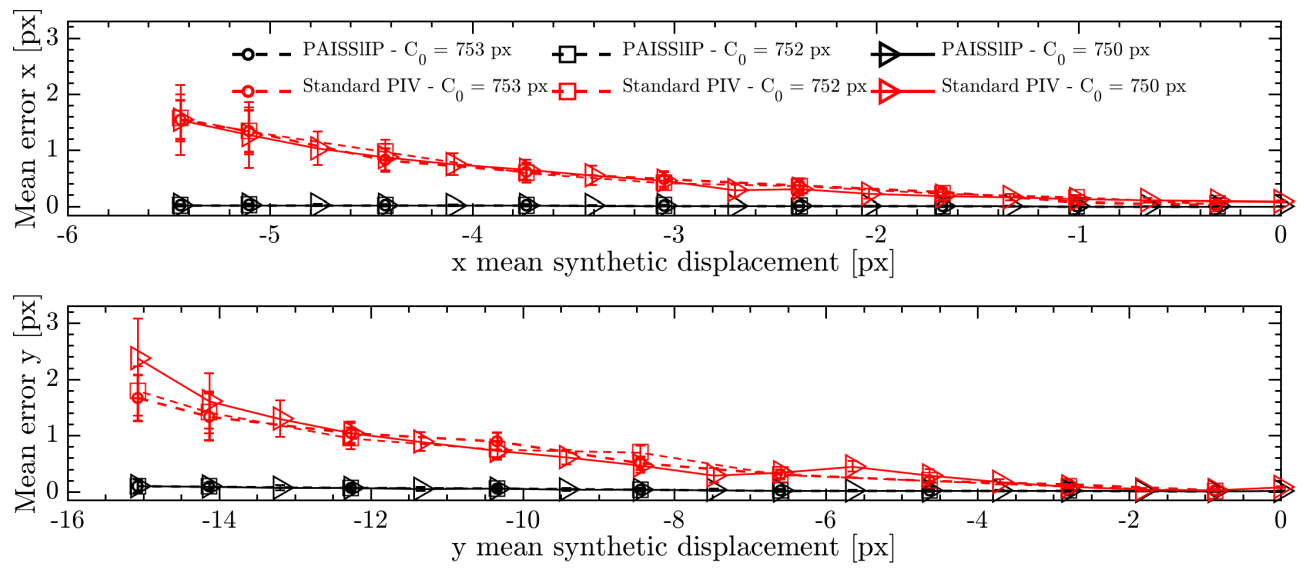

Figure 13: Mean discrepancies of the $\mathrm{x}$ and $\mathrm{y}$-displacements for $0.6 \cdot A_{Y}$ but shifted interface location in the images.

revealed by the dependence of the error on the interface position, whereas PAISSIIP remains completely unaffected, thus proving enhanced robustness.

\section{Experimental Assessment}

The experimental assessment of PAISSIIP has been done by measuring the velocity field of water contained in a cylindrical vessel, excited by a small amplitude sinusoidal wave (see Fig.14). This specific configuration was chosen because of the availability of related simplified theoretical models both for the description of the different sloshing modes and associated amplitudes $\eta$, as well as the large number of corresponding applications.

\subsection{Experimental Setup and Test Matrix}

A schematic of the experimental facility used to characterize water sloshing by means of Fluorescent Particle Image Velocimetry is shown in Fig. 15a. 


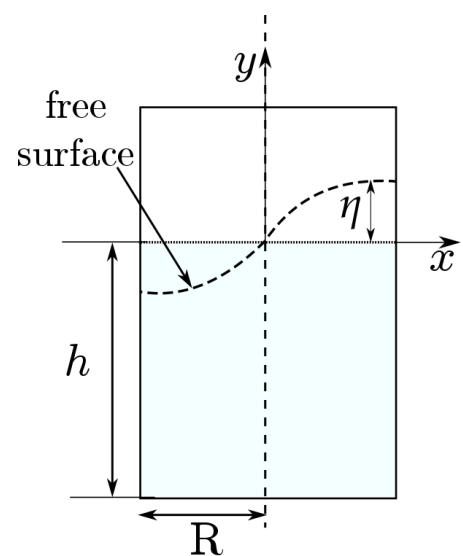

Figure 14: Container-fluid system: circular cylindrical vessel filled till a fixed level h: example of free surface displacement.

The principal element of the experimental setup was the sloshing cell of which the main component consisted of a cylindrical pipe placed inside a rectangular cuboid (see Fig. 15c). The pipe had an inner radius $R$ of 40 $\mathrm{mm}$ and a height $H=150 \mathrm{~mm}$. Both the pipe and cuboid were made of Plexiglas and the volume between the two elements was filled with water to achieve a partial refractive index matching and therefore to minimize optical distortions. The cell was fixed to a sloshing table able to produce sinusoidal motions with amplitudes ranging from 1 to $10 \mathrm{~mm}$ and frequencies from 0.1 to $6.6 \mathrm{~Hz}$ with an accuracy of $0.02 \mathrm{~Hz}$. The position of the table was recorded by an Optical Displacement Sensor (ODS) with an accuracy of $18 \mu \mathrm{m}$. The ODS signal was also used as input to perform phase locked image acquisitions with a selected phase of the sinusoidal motion. The complete optical arrangement is presented in Fig. 15b and included, in addition to the standard PIV optics, a high pass filter with low cut off wavelength $\lambda_{\text {cut }}=580$ 


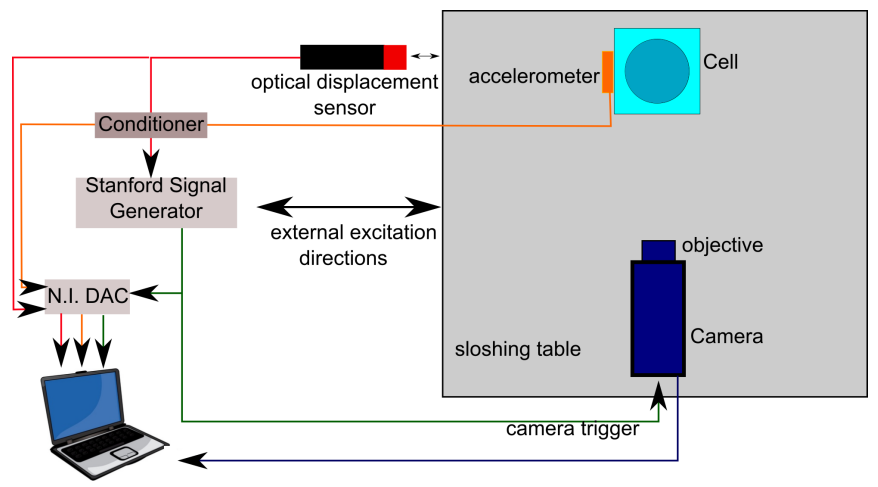

(a)

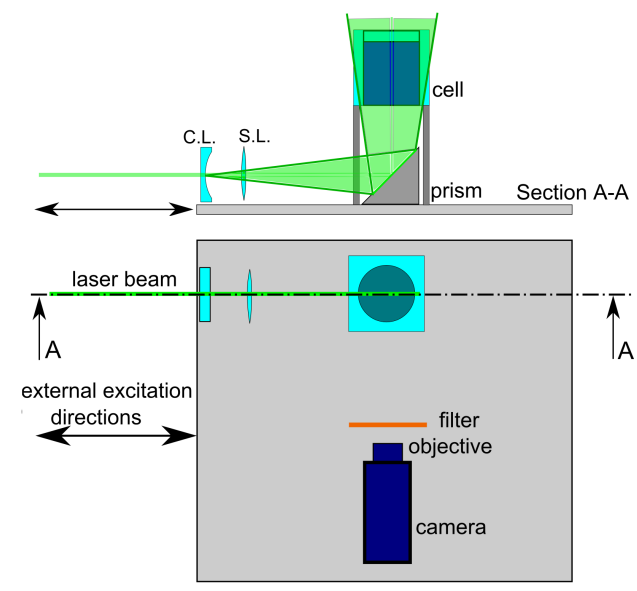

(b)
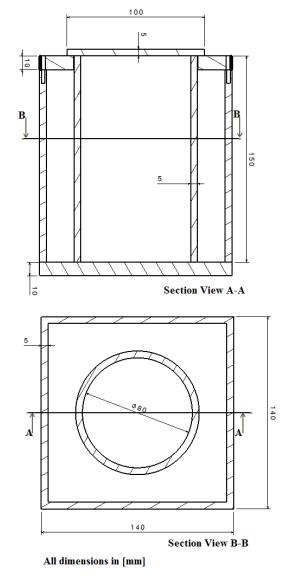

(c)

Figure 15: (a) Schematic of the experimental facility. (b) Optical arrangement. (c) Sloshing cell.

$\mathrm{nm}$ to collect the light emitted by the fluorescent seeding particles. A double pulsed 250mJ Nd:Yag laser was used to illuminate the tracer particles. PIV images were recorded by a double frame camera with a sensor of $1376 \times 1040$ $\mathrm{px}^{2}$ and a $35 \mathrm{~mm}$ Nikkor objective $(\mathrm{F} / 1.8)$ yielded a resolution of 0.1045 $\mathrm{mm} / \mathrm{px}$. Imposed separation times $\Delta t$ depended on the external phase motion and were such that a maximum particle displacement of the order of 10 
Table 3: Initial time $t_{a}$ for the first snapshot acquisition and delay time $\Delta t$ for the second image snapshot relative to the phase locked acquisition of Fig. 16.

\begin{tabular}{ccccccccccc} 
& $\mathrm{T} 1$ & $\mathrm{~T} 2$ & $\mathrm{~T} 3$ & $\mathrm{~T} 4$ & $\mathrm{~T} 5$ & $\mathrm{~T} 6$ & $\mathrm{~T} 7$ & $\mathrm{~T} 8$ & $\mathrm{~T} 9$ & $\mathrm{~T} 10$ \\
\hline$\Delta t[\mathrm{~ms}]$ & 80 & 99 & 90 & 90 & 80 & 99 & 99 & 80 & 70 & 60 \\
$t_{a}[-]$ & 0.09 & 0.19 & 0.29 & 0.39 & 0.49 & 0.59 & 0.69 & 0.79 & 0.89 & 0.99 \\
\hline \hline
\end{tabular}

Table 4: Properties of fluorescent particles.

\begin{tabular}{cccc}
\hline \hline $\begin{array}{c}\text { Diameter } \\
{[\mu \mathrm{m}]}\end{array}$ & $\begin{array}{c}\text { Density } \\
{\left[\mathrm{kg} \mathrm{m}^{-} 3\right]}\end{array}$ & $\begin{array}{c}\text { Absorption band } \\
{[\mathrm{nm}]}\end{array}$ & $\begin{array}{c}\text { Emission peak } \\
{[\mathrm{nm}]}\end{array}$ \\
\hline \hline $10-45$ & 998 & $460-650$ & 605 \\
\hline
\end{tabular}

pixels at the interface was obtained to retrieve an adequate dynamic velocity range. The values used are reported in table 3 . The reader is reminded that such magnitudes in interface translation (and consequently deformation) inhibit the use of a unique curvilinear coordinate transformation as advocated in section 2 and warrants the novel image processing introduced within the current work.

The properties of the fluorescent particles used are summarized in Table 4. The Stokes number relative to the test performed was $\mathcal{O}\left(10^{-4}\right)$, indicating these particle to be suitable tracers. It is important to underline that since the density of the particles was slightly smaller than that of water, during the filling of the cell a small quantity of particles was trapped at the liquid/gas interface. The presence of these particles allowed the detection of the liquid/gas interface and therefore facilitated the dynamic masking of the images [?]. 
The experiments were performed in so-called "deep water" conditions with water level to pipe radius ratio $h / R=2.4$ (cf. Fig.14). The vessel was excited by a sinusoidal wave with amplitude $X_{0}=1.2 \mathrm{~mm}$ and a frequency $F=2 \mathrm{~Hz}$. This frequency was sufficiently below the first natural frequency of the system $\left(f_{n}=3.38 \mathrm{~Hz}\right)$ and the ensuing sloshing could therefore be considered as belonging to the linear regime [? ]. PIV measurements were performed in stationary conditions for 10 different phases of the excitation wave (see Fig. 16).

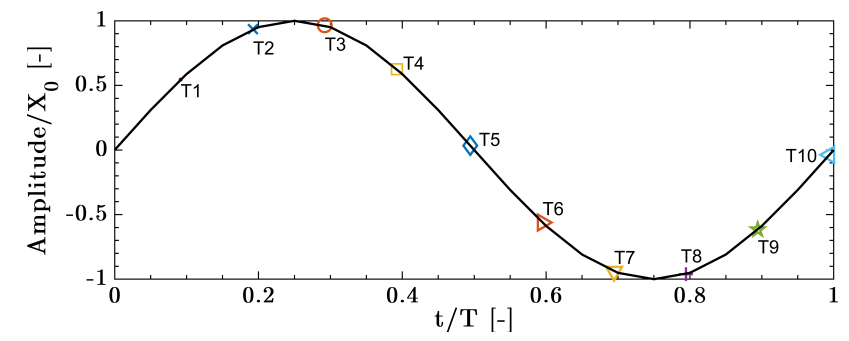

Figure 16: Synchronization of the phase locked acquisitions.

\subsection{Data Analysis and Results}

\subsubsection{Spatial profiles}

The PIV images were analyzed with both PAISSIIP and the standard PIV code adopting the parameters reported in Table 2. In each image the gas-liquid interface was detected using a Radon transform based algorithm [? ] (Fig. 17), followed by a dynamic image masking to exclude the non-seeded gas phase in the PIV recordings from the image analysis.

For each phase locked position, the velocity profiles along the liquid height at radial locations $x=-R / 2$ and $x=R / 2$ obtained by both PAISSIIP and 


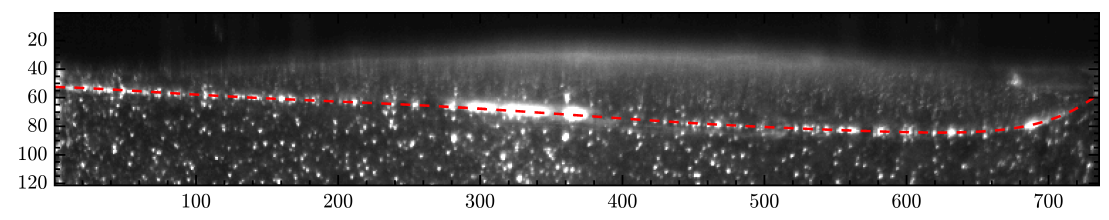

Figure 17: Detection of the interface for phase locked acquisitions, case $\mathrm{F}=2 \mathrm{~Hz}$.

the standard PIV algorithm were compared and are presented in Fig. 18. Here the obtained velocity fields from the two codes have been interpolated pixelwise, selecting only data pertaining pixels located below the original vector locations nearest to the interface. The reader is reminded that the zero $y$-coordinate corresponds to the position of the interface at rest.

As predicted by theory [? ] it is possible to observe that for both cases the decay of the $\mathrm{x}$ and $\mathrm{y}$ velocity components is exponential. Nevertheless, even if the profiles obtained with the two algorithms look extremely similar, they strongly differ in the region immediately below the liquid/gas interface where the standard PIV algorithm occasionally predicts unphysical velocity gradients as illustrated in Fig. 19. The failure of the standard code is expected to be even more evident in case of higher gradients or improved spatial resolution in the experiment.

\subsubsection{Temporal velocity profiles}

The time evolution of both velocity components obtained with PAISSIIP at a location $x=-R / 2$ and $0.5 \mathrm{~mm}$ ( 5 pixels) below the instantaneous experimental liquid/gas interface is reported in Fig. 20 (black bars). Herein also the instantaneous velocity components obtained by the linear sloshing theory [? ] are presented for each phase locked position corresponding to the first frame snapshot (red crosses). As expected, both velocity components 


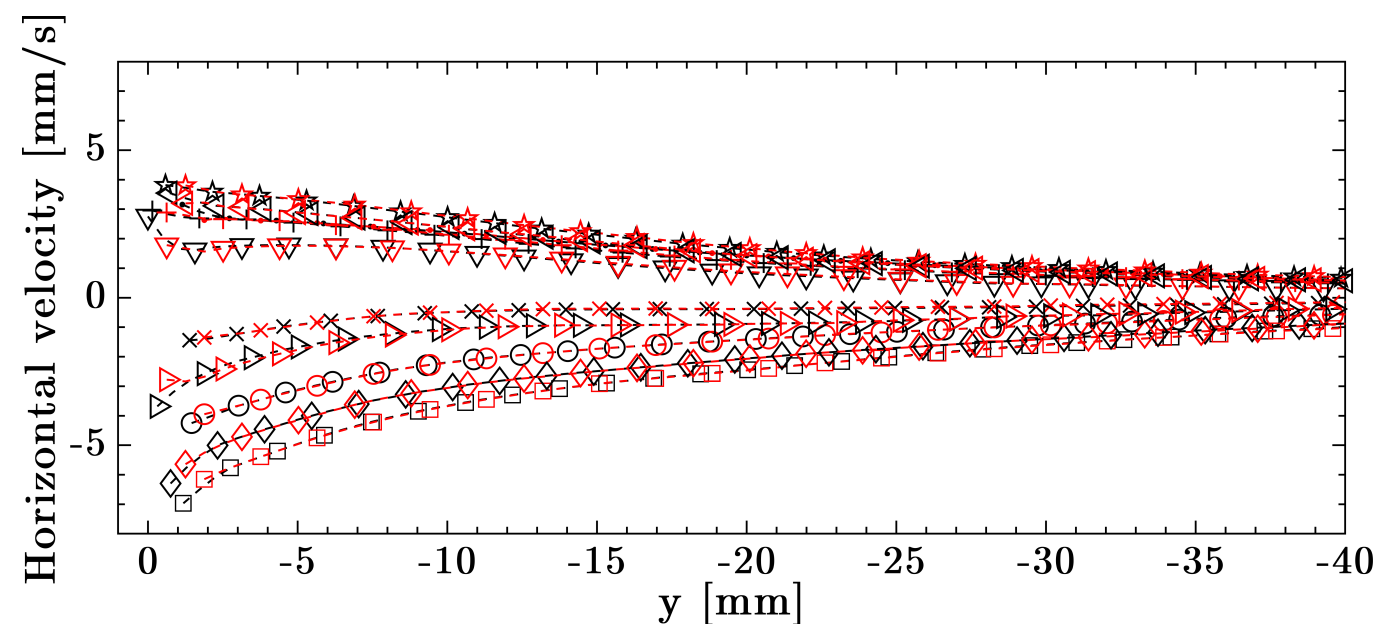

(a)

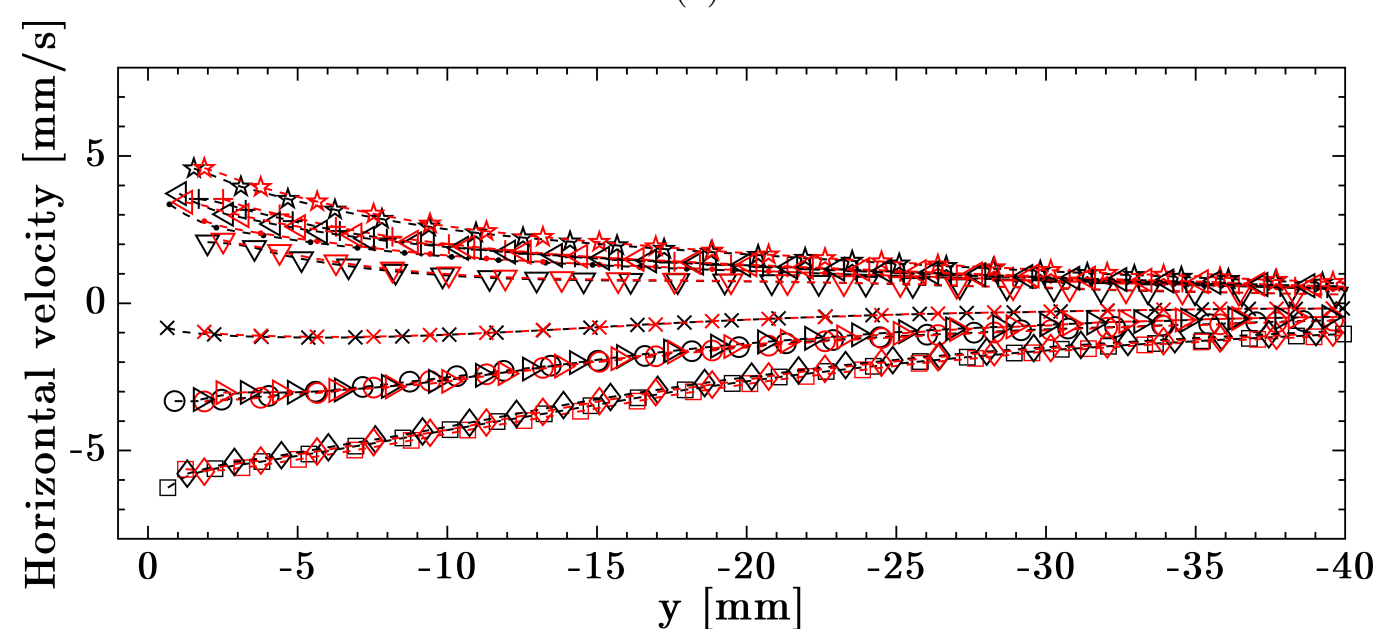

(b) 


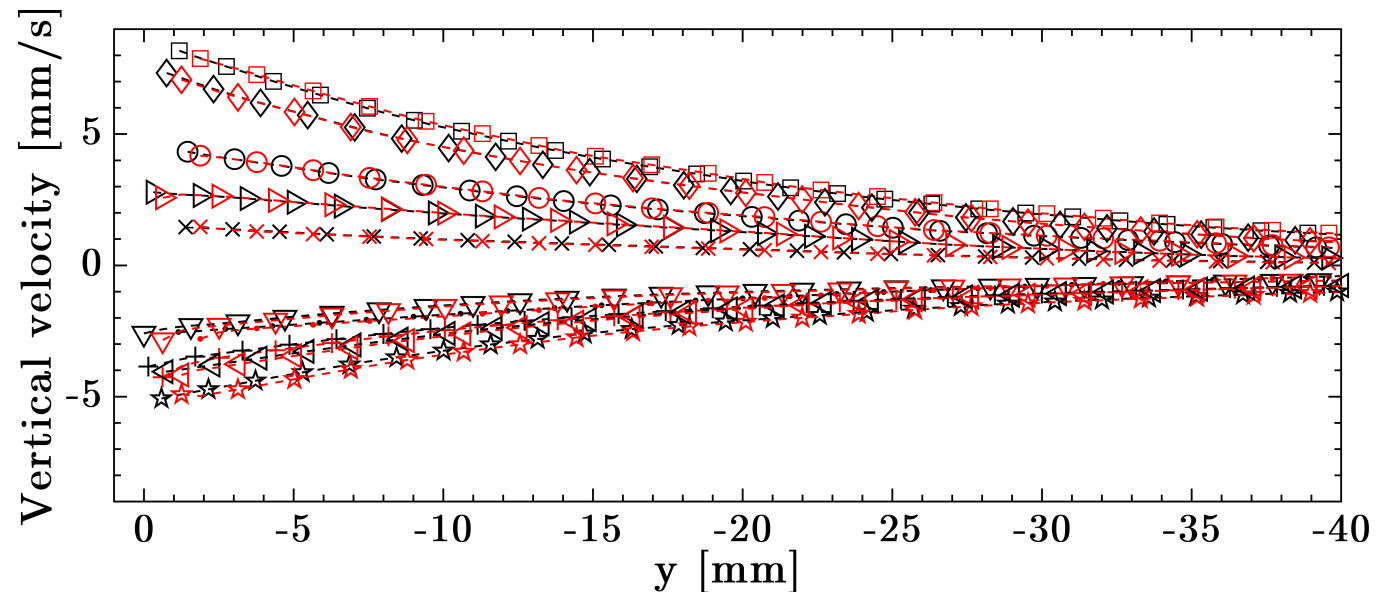

(c)

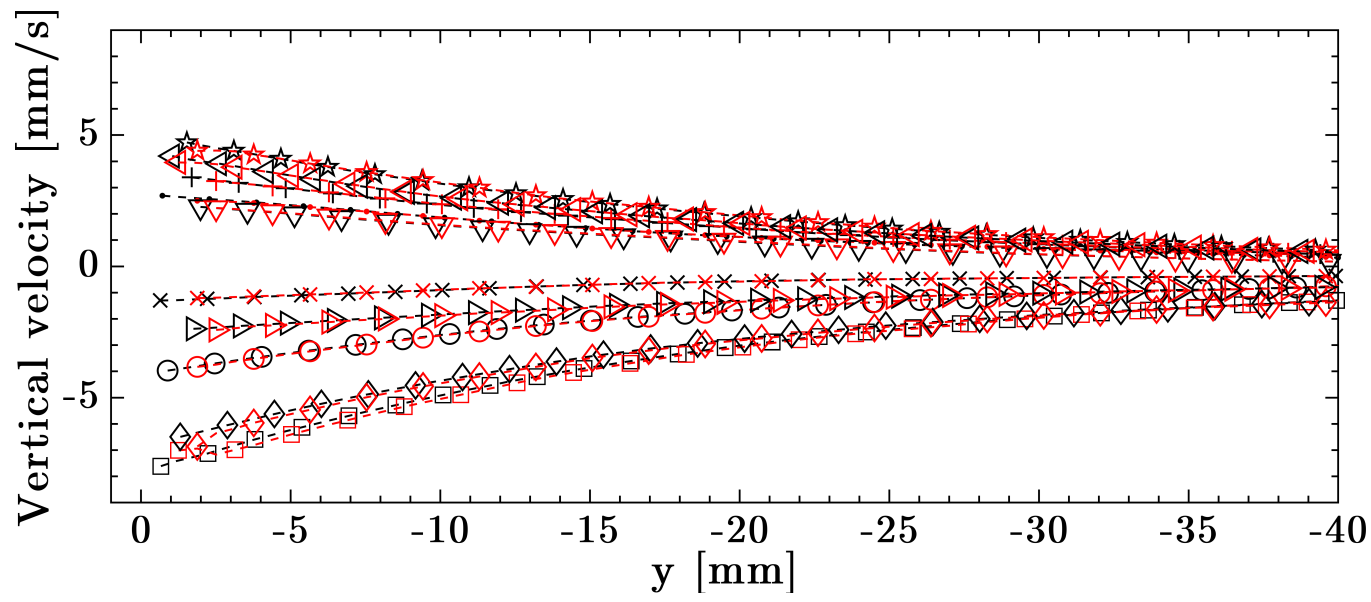

(d)

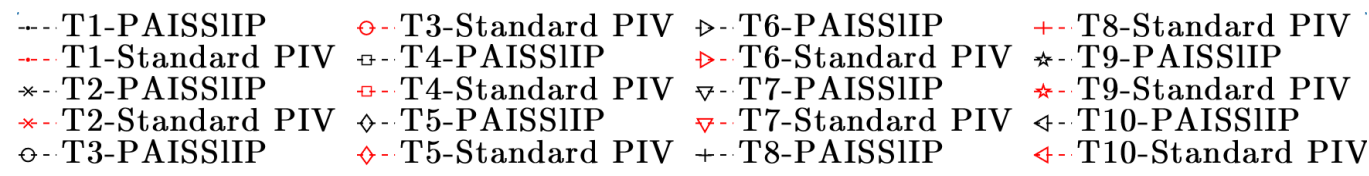

Figure 18: Vertical profiles of the velocity components in two symmetrical radial positions: (a) horizontal velocity at $x=-0.5 R$; (b) horizontal velocity at $x=0.5 R$; (c) vertical velocity at $x=-0.5 R ;$ (d) vertical velocity at $x=0.5 R$. The obtained velocity fields from the two codes have been reinterpolated on the same Cartesian grid. Only displacements attributed to pixels located below those vectors which were nearest to the interface are considered. To enhance readability, markers have been undersampled by a factor 3 . 


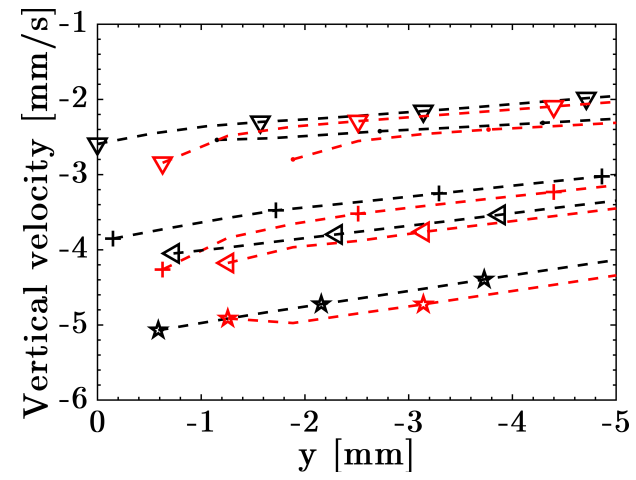

(a)

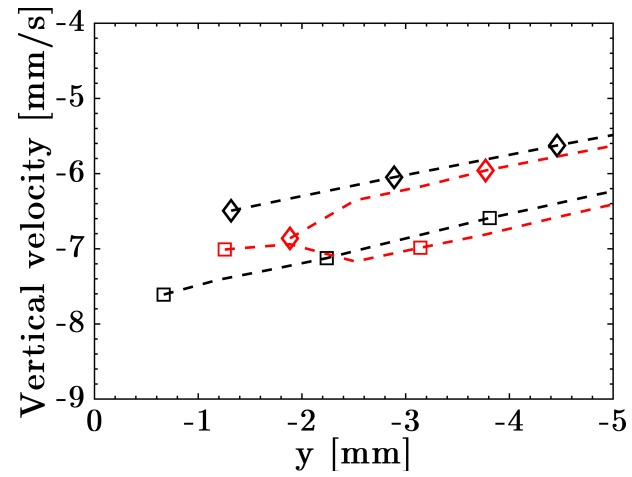

(b)

Figure 19: Close-up of the profiles presented in Fig. 18c and Fig. 18d; (a) $x=-0.5 R$; (b) $x=0.5 R$. The legend is as per Fig. 18 .

vary as $\cos (t / T)$ but a certain phase delay can be observed with respect to the instantaneous theoretical velocity profiles, which is considered in phase with the external velocity applied [? ]. This phase delay is due to two contributions. First, the response delay of the system as already observed by [? ] due to viscous dissipation neglected by the linear theory. Second, PIV inherently measures the displacement of particle patterns between frames, i.e. the particles' start and end position and therefore not an instantaneous but integrated velocity. To check the origin of the inherent time delay observed in the PIV data, the linear theory is used to calculate the location of a particle after the delay $\Delta t$ (tab. 3) when the particle is initially placed (time $t_{a}$, cf. tab. 3) at the corresponding experimental coordinates. The resulting displacement vectors are shown in figure 20 as blue bars. Considering the time integration, a better agreement between the linear theory and PIV data is obtained. Linear theory generally overestimates the sloshing amplitudes though by up to 40 a\%, which is attributed to the neglect of viscosity. 
This experimental assessment thus leads to two additional findings; (1) linear sloshing theory is incomplete in so far that it should include viscosity, (2) PIV can be used to extract velocity data, yet one must account for the fact that between image recordings the velocity field changes while PIV yields an integrated velocity. It is therefore incorrect to directly compare PIV data with instantaneous numerical data when dealing with unsteady flow fields and sufficiently large particle image displacements. This observation is of particular importance when using PIV to validate numerical simulations of sloshing as the latter must first be integrated over the PIV image separation time $[?]$.

\section{Conclusions}

In this work an improved image processing tool for wavy free surface flows is presented. Standard interrogation routines have been argued to become unreliable in the presence of too strong interface motion and sub-optimal vector density. Also the use of image mapping is discouraged due to the ambiguity in the required Jacobian transformations. To this extent the proposed algorithm incorporates adaptive image sampling, thereby placing more vectors in the vicinity of the interface. In addition, images are processed in sequential sub-domains where correlation windows are progressively reduced in size towards the interface. In the final processing stage image projection is applied based on only one detected interface outline and an alternative interface gradiometry approach has been implemented in view of the need for higher resolution and accuracy at wavy interfaces. The attainable improvements have been corroborated by a numerical assessment based on synthetic 


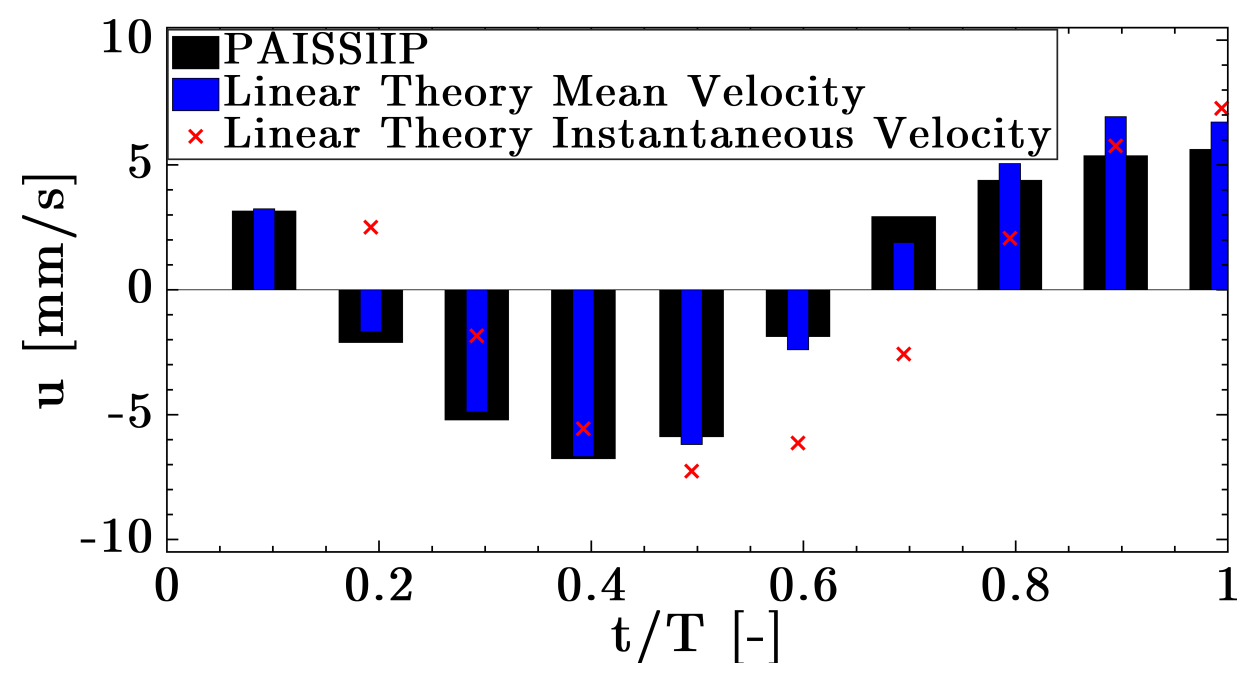

(a)

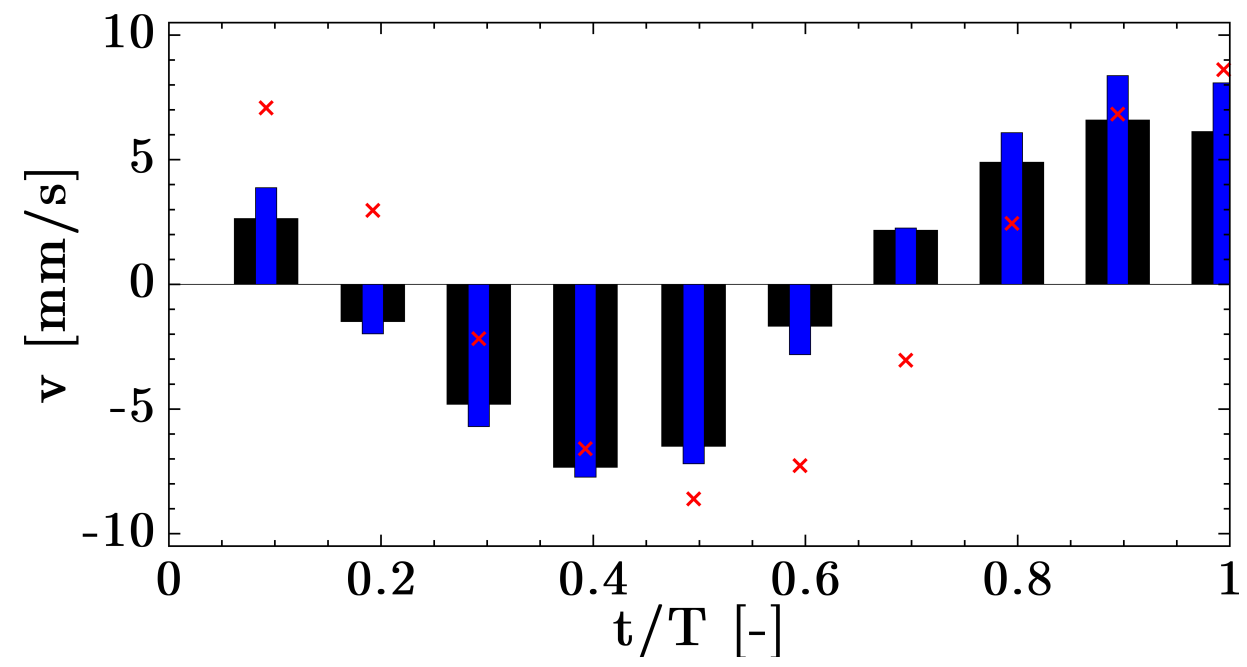

(b)

Figure 20: Comparison between the velocity components obtained by means of PAISSIIP (black bars) and the velocity obtained by using the linear theory from [? ] at the same $x$-coordinate position $x=-R / 2$ and 5 pixels below the instantaneous experimental interface position of the first image snapshot. The linear theory is used to calculate the instantaneous velocity (red crosses) at the instant $t_{a}$ (tab. 3) and the end position of the particles after the delay time $\Delta t$ (tab. 3 ) from which is calculated the mean velocity (blue bars). (a) $u$-component; (b) $v$-component. 
images where the independence of the adaptive methodology with respect to interface curvature was highlighted, contrary to the standard approach. In addition, a drastic reduction in overall displacement error from approximately 2 pixels to less than 0.2 pixels was observed, with a simultaneous drastic reduction in data scatter. A standard PIV code and the proposed approach have been used on experimental PIV images of liquid sloshing. It has been shown that while the conventional image processing routine yields unphysical gradients in velocity near the liquid/gas interface, the proposed routine adopting image projection and adaptive sampling produced reliable results. Juxtaposing the experimental data with linear theory, the disregard of viscosity in the latter was shown to be a limiting factor. Moreover, it was shown that the dynamic (temporal) behaviour of the underlying velocity field must be appropriately accounted for when assessing numerical data based on PIV results.

\section{Appendix}

Considering interfaces at times $t_{1}$ and $t_{2}$ with a horizontal reference line selected halfway between the boundaries. Pseudo-images are thus aligned on a common horizontal line located in the original pixel reference frame at $y_{r e f}$ (Fig. 21). Referencing the interface location by $y_{\text {int }}$, a pixel location at $y$ will be shifted to a new location

$$
y_{n}=y+\left(y_{r e f}-y_{\text {int }}(x)\right) \cdot \Psi\left(\left|y-y_{\text {int }}(x)\right|\right)
$$

Such translations should however only be local and subside away from the interface, which is controlled by the function $\Psi(\cdot)$. A suitable function is 


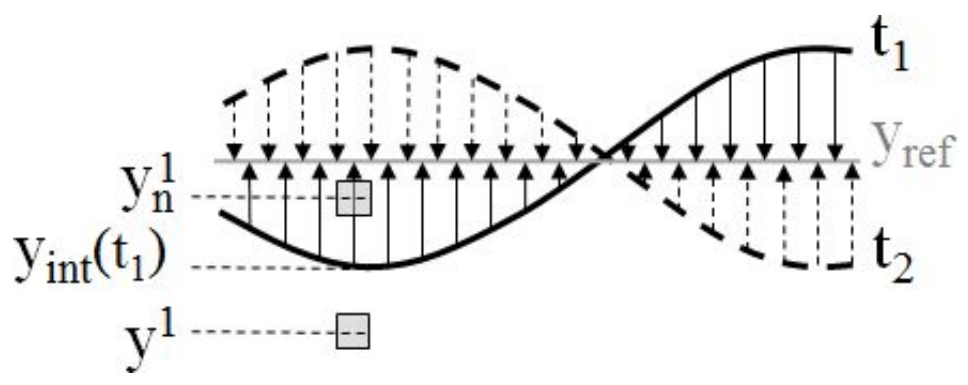

Figure 21: Principle of image shifting: pixels are relocated to a new position $y_{n}^{1}$ in the direction of a reference location $y_{r e f}$. The shift is proportional to the original distance from the interface $y_{\text {int }}$.

therefore the Wendland radial basis function $\varphi(r, \epsilon)$;

$$
\begin{gathered}
\varphi=(1-\epsilon r)_{+}^{4}(4 \epsilon r+1) \\
\frac{d \varphi(r, \epsilon)}{d r}=-20 \cdot \epsilon^{2} \cdot r \cdot(1-\epsilon r)_{+}^{3}
\end{gathered}
$$

where $\epsilon$ defines the extent of the imposed evolution, $(x)_{+}=x$ if $x \geq 0$ and 0 else, and $r$ signifies the radial distance from the detected interface. To avoid mesh folding pixel sequences must remain unchanged, i.e. $d y_{n} / d y>$ 0. Utilizing the derivative of the Wendland function 17 and noting that $(1-\epsilon r)^{3}<0.12$ for $\epsilon r \leq 1$, an upper limit on $\epsilon$ can be established: $\epsilon<$ $\left(2.4 \cdot\left|y-y_{\text {int }}\right|_{\max }\right)^{-1}$. In the transformed images, standard interrogation processes are subsequently applied as per convention.

Despite intrinsically simple and quick, this methodology introduces elongated particle images and artificial gradients; let a particle at time $t_{1}$ be located at $\left(x^{1}, y^{1}\right)$. Imposing a constant displacement $\mathrm{v}$ along the $\mathrm{y}$-direction, the position at time $t_{2}$ is given as $\left(x^{1}, y^{2}\right)=\left(x^{1}, y^{1}+v\right)$. The distance between 
the particle images in the pseudo images can be easily shown to given by

$$
y_{n}^{2}-y_{n}^{1}=v \cdot\left(1-\Psi\left(\left|y^{1}-y_{\text {int }}(x)\right|\right)\right)
$$

Since $\Psi$ varies spatially, the distance between two particles in the transformed images will no longer be constant, but vary depending on their original location.

To exemplify particle images with a uniform displacement of 20 pixels in vertical direction have been generated and analyzed adopting image shifting. Fig. 22a shows an exemplary synthetic image while Fig.22b depicts the average $\beta_{v}$ and rms error $\sigma_{v}$ in vertical displacement. As a result of the introduced curvature, errors in the order of the imposed displacement are found within 100 pixels from the interface. Clearly the use of pixel shifting is discouraged.

\section{Acknowledgments}

Alessia Simonini is supported by a FRIA grant from the Belgian FNRS. FNRS and VKI-AA travel grant are also acknowledged for funding the expenses of Ms. Simonini as visiting $\mathrm{PhD}$ in Bristol University. 


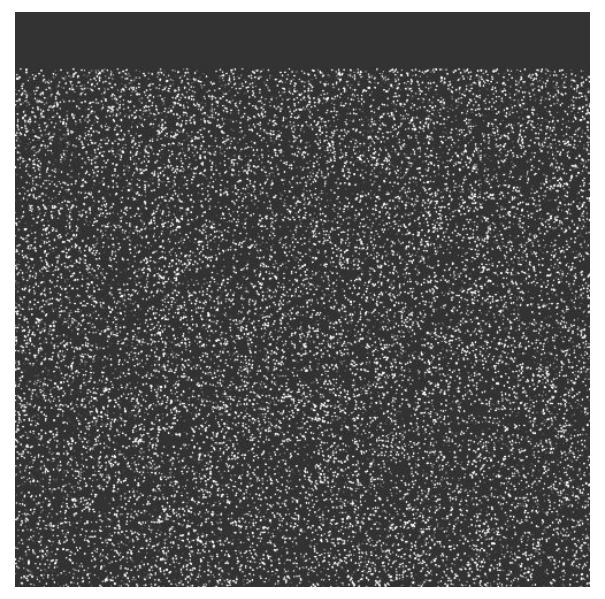

(a)

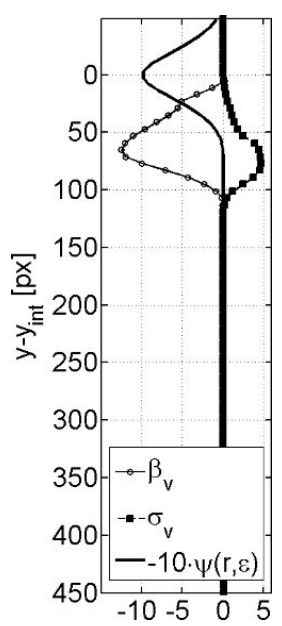

(b)

Figure 22: Example of displacement gradients artificially introduced by pixel shifting (a) PIV synthetic image of an uniform vertical motion (b) Error in the vertical displacement as function of distance from the interface. 